\title{
Purity Profiling of Peptide Drugs
}

\section{Sylvia Van Dorpe, Mathieu Verbeken, Evelien Wynendaele and Bart De Spiegeleer*}

Drug Quality and Registration (DruQuaR) group, Faculty of Pharmaceutical Sciences, Ghent University, Harelbekestraat 72, B-9000 Ghent, Belgium

\begin{abstract}
The quality of a peptide drug mainly depends on its impurity profile, with the emphasis on the related impurities. These impurities may be biomedically active, alter the desired efficacy or induce unwanted toxicity, an aspect which is termed the "functional quality" of the peptide drug. Therefore, regulatory authorities have set up guidances or have legally established specification limits to assure a consistent purity of these peptide drugs. For the active pharmaceutical ingredients (APIs), the pharmacopoeial monographs are legally binding. Additional information can be found in regional and international guidelines. For the finished pharmaceutical drug products (FDPs) containing peptide active ingredients, only general guidelines are available. The construction of a complete related-impurity profile is very challenging due to the wide availability of different protecting groups, coupling agents and additives that may be used during peptide synthesis. In addition, chemical degradation, occurring during synthesis, formulation or at storage, may occur as well, including not only so-called pure chemical degradation but interaction with excipients as well. This review provides an update of the regulatory and scientific rationales behind the related impurities in peptide drugs.
\end{abstract}

Keywords: Peptides;Related impurity;Degradation;Synthesis;Excipi ent interaction; Functional quality; Specification limits.

Abbreviations: 1-Ada: 1-Adamantyl; 2-Cl-Trt: 2-Chlorotrityl; 2-Ph-iPr: 2-Phenylisopropyl; Acm: Acetamidomethyl; Al: Allyl; Alloc:Allyloxycarbonyl; API: Active pharmaceutical ingredient; Azoc: Azidomethoxycarbonyl; BHA: Benzhydrylamine resin; BHT: 2,6-dit-butyl-4-methylphenol; Bis-tBoc: $\quad \omega, \omega$ '-bis-tert-Butyloxycarbonyl; Bn: Benzyl; tBoc-NMEC: tBoc-N-methyl-N-[2-(methylamino)ethyl] carbamoyl; Bom: N-Benzyloxymethyl; BOP: O-Benzotriazol-1yloxytris(dimethylamino)phosphoniumhexafluorophosphate; BrBn: 2-Bromobenzyl; BrZ: 2-Bromobenzyloxycarbonyl; cHx: Cyclohexyl; Cl-Z: 2-Chlorobenzyloxycarbonyl; Cpd: Cyclopropyldimethylcarbinyl; CZE: Capillary zone electrophoresis; Dcb: 2,6-Dichlorobenzyl; DCC: Dicyclohexylcarbodiimide; Ddiv: 1-(4,4-dimethyl-2,6-dioxocyclohex-1-ylidine)-3-methyl-butyl; Dmab: 4-(N-[1-(4,4-dimethyl2,6-dioxocyclohexylidene)-3-methylbutyl]-amino)benzyl; Dmbz: 2,4-Dimethoxycarbonyl; Dmnb: 4,5-Dimethoxy-2-nitrobenzyl; dNBS: 2,4-Dinitrobenzenesulfonyl; Dnp: N-2,4-Dinitrophenyl; Dnpe: 2-(2,4-Dinitrophenyl)ethyl; Doc: N-2,4-Dimethylpent-3-yloxycarbonyl; Dts: Dithiasuccinoyl; DVB: Divinylbenzene; FDP: Finished drug product; Fmoc: 9-fluorenylmethoxycarbonyl; Fmoc-Osu: Fmoc-oxysuccinimide; For: Formyl; GLP: Good laboratory practice; GMP: Good manufacturing practice; HATU: O-(7-Azabenzotriazol-1-yl)-1,1,3,3tetramethyluronium hexafluorophosphate; HBTU: Hexafluorophosphate; HFA: Hexafluoroacetone; Hmb: N-(2-hydroxy-4-methoxybenzyl); HOAt: 1-Hydroxy-7-azabenzotriazole; HOBt: 1-Hydroxybenzotriazole; Hoc: Cyclohexyloxycarbonyl; HONB: N-hyddroxy-5-norbornene-2,3-dicarboximide; HOSu: N-Hydroxysuccinimide; HPLC: High-performance Liquid Chromatography; IP: Indian Pharmacopoeia; ivDde: 1-(4,4-dimethyl-2,6-dioxocylcohex-1-ylidene)-3-methylbutyl; MA: Marketing authorization; Mbh: 4.4'-Dimethoxybenzhydryl; MBHA: Methylbenzhydrylamine resin; MDD: Maximum daily dose; Meb: p-Methylbenzyl; Men: $\beta$-Menthyl; MeSub: 2-Methoxy-5dibenzosuberyl; MIS: 1,2-Dimethylindole-3-sulfonyl; Mmt: Monomethoxytrityl; MNPPOC: 2-(3,4-Methylenedioxy-6-nitrophenyl) propyloxycarbonyl; Mob: p-Methoxybenzyl; Mpe: $\beta$-3-Methylpent3-yl; Msc: 2-(methylsulfonyl)ethoxycarbonyl; Mtr: 4-Methoxy-2,3,6trimethylphenylsulfonyl; Mts: Mesityl-2-sulfonyl; Mtt: 4-Methyltrityl;
Nin: Ninhydrin; Nmbu: 4-(N-methylamino)butanoyl; NMR: Nuclear Magnetic Resonance; $\mathrm{NO}_{2}$ : Nitro; NPPOC: 2-(2-Nitrophenyl)propyloxycarbonyl; Npys: 3-Nitro-2-pyridinesulfenyl; NVOC: 4-nitroveratryloxycarbonyl; OBzl: Oxybenzyl; oNB: o-Nitrobenzyl; oNBS: oNitrobenzenesulfonyl; oNZ: o-nitrobenzyloxycarbonyl; OtBu: oxytertbutyl; Pbf: 2,2,4,6,7-Pentamethyl-2,3-dihydrobenzofuran-5-sulfonyl; PEG: Polyethyleneglycol; Pen: 3-Pentyl; PG: Protecting group; PhAcm: Phenylacetamidomethyl; Phdec: Phenyldisulphanylethyloxycarbonyl; Ph. Eur.: European Pharmacopoeia; Ph. Int.: International Pharmacopoeia; PLA: poly(lactic acid); PLG: poly(D,L-lactide-co-glycolide); PLGA: poly(D,L-lactic acid-co-glycolic acid); Pmbf: 2,2,4,6,7-Pentamethyl-5-dihydrobenzofuranylmethyl; Pmc: 2,2,5,7,8-Pentamethylchroman-6-sulfonyl; pNB: p-Nitrobenzyl; pNZ: p-nitrobenzyloxycarbonyl; Poc: Propargyloxycarbonyl; PS: Polystyrene; PTMSE: (2-Phenyl-2trimethylsilyl)ethyl; PyBOP: O-Benzotriazol-1-yloxytris(pyrrolidino) phosphoniumhexafluorophosphate; Pydec: 2-Pyridyldisulphanylethyloxycarbonyl; QbD: Quality by Design; SPPS: Solid-phase peptide synthesis; S-Pyr: 2-Pyridinesulfenyl; StBu: 5-tert-Butylmercapto; Sub: 5-Dibenzosuberyl; Suben: 5-Dibenzosuberenyl; TBDMS: tert-Butyldimethylsilyl; TBDPS: tert-Butyldiphenylsilyl; tBoc: tert-Butyloxycarbonyl; TBTU: O-Benzotriazol-1-yloxytris-1,1,3,3-tetramethyluronium tetrafluoroborate; tBu: tert-butyl; TCP: Tetrachlorophthaloyl; TEGBz, TEGBn: 4-(3,6,9-Trioxadecyl)oxybenzyl; tfa:Trifluoroacetyl; TLC: Thin layer chromatography; Tmob: 2,4,6-Trimethoxybenzyl; Tos: p-Toluenesulfonyl; Troc: 2,2,2-trichloroethyloxycarbonyl; Trt: Trityl; USP: United States Pharmacopoeia; Xan: 9-Xanthenyl; Z:Benzyloxycarbonyl

*Corresponding author: Bart De Spiegeleer Drug Quality and Registration (DruQuaR) group, Faculty of Pharmaceutical Sciences, Ghent University, Harelbekestraat 72, B-9000 Ghent, Belgium, Tel.: +32 9264 8100; Fax: +32 9264 8193; E-mail: Bart.DeSpiegeleer@UGent.be

Received October 22, 2011; Accepted November 10, 2011; Published November 12, 2011

Citation: Van Dorpe S, Verbeken M, Wynendaele E, De Spiegeleer B (2011) Purity Profiling of Peptide Drugs. J Bioanal Biomed S6: 003. doi:10.4172/1948-593X.S6003

Copyright: (c) 2011 Van Dorpe S, et al. This is an open-access article distributed under the terms of the Creative Commons Attribution License, which permits unrestricted use, distribution, and reproduction in any medium, provided the original author and source are credited. 


\section{Introduction}

Peptides are becoming key players in the pharmaceutical industry, gaining more and more attention as possible diagnostics or therapeutics. Worldwide, more than 60 peptides are marketed, 270 are in clinicalphase testing and 400 in advanced preclinical phases. It is thus obvious that peptides show a great promise as drugs [1]. Although peptides demonstrate a high biological activity in combination with low toxicity and high specificity compared to small molecules, they suffer from low stability, low oral bioavailability and delivery issues [1-4]. They cannot be considered as conventional small organic molecules because of their building-blocks system, relative large molecular weight and physicochemical characteristics, requiring specific ways of synthesis and care in the formulation. This handling complexity may result in related byproducts, which are likely to be biologically active. Since the safety of a drug product is dependent not only on the toxicological properties of the active drug substance, but also on the toxicological properties of its impurities, there is an ever-increasing concern over these impurities present in active pharmaceutical ingredients (APIs) and finished drug products (FDPs) $[5,6]$.

Impurities may be process- or product-related, originated during synthesis, manufacturing, or storage and should be characterized to the necessary extent. For example, in the discovery phase, they are also to be evaluated for their biological activity in order to avoid false positives or negatives due to the presence of these impurities [7-10]. This step is crucial in the evaluation of biomedical activity since these impurities may be more active compared to the main component or even alter activity.

Impurity profiling (i.e. the identity as well as the quantity of impurities in the pharmaceutical drug) is now gaining critical attention from regulatory authorities in order to assure and control the quality. The different Pharmacopoeias, such as exemplified by the European Pharmacopoeia (Ph. Eur), United States Pharmacopeia (USP), International Pharmacopoeia (Ph. Int.) and Indian Pharmacopoeia (IP) are defining specification limits for reporting, qualification and identification of impurities present in the APIs or FDPs formulations, based upon found levels in approved market samples [11-14].These principles are based on the ICH guideline Q3A(R), which stipulates different thresholds or action limits based upon the maximum daily dose (MDD).

Next to the presence of synthetic impurities, peptide substances or finalized products may be chemically unstable. Degradants are produced already during synthesis, purification or manufacturing, but mainly during storage.

\section{Regulatory framework}

From a regulatory quality perspective, several texts have to be considered in setting proper impurity specifications against which the quality of peptide drugs is evaluated with the final purpose to make a release decision. Releasing a pharmaceutical product implies that the quality is sufficiently high and consistent, as justified in the approved marketing authorization (MA) dossier, so that the probability of unwanted and unexpected harm to the patient is minimised. At a global level, the ICH quality guidelines, adopted by the European EMA, the US FDA and the Japanese PMDA and widely followed by the rest of the world, including the WHO, describe how to deal with impurities and specification settings in an international regulatory environment, i.e. sensu stricto only at the phase of preparing the submission of a MA application [15-17]. These guidelines are to be combined with regional laws and guidelines, as well as the regional and/or national pharmacopoeia. Recently, the Ph. Eur. has extended its mandatory general monograph "Substances for pharmaceutical use" [18]with an explanatory general chapter 5.10 "Control of impurities in substances for pharmaceutical use" [19]. While these are sensu stricto only applicable to substances explicitly monographed in the Ph. Eur., the analytical procedures and/or acceptance criteria described therein are clearly a rational basis for other drug substances and drug products not described in the Ph. Eur. as well $[5,6]$.

All Ph. Eur. monographs, but not the general chapters, are legally binding as stated in Directive 2001/83/EC [20]. The Ph. Eur. as well as the other pharmacopoeia give limits of impurities in its API-specific monographs, based upon the impurity profiles found in APIs of products already on the market and thus in clinical use. Therefore, the APIspecific pharmacopoeialmonograph reflects the current status of an API quality. Besides the API-specific monographs, there are the mandatory general monographs which need to be combined with the API-specific monograph. If this specific or general monograph is explicitly referring to a general chapter, then this general chapter becomes mandatory as well. Hence, for example, the specific monograph of buserelin API [21] is to be combined with the general monograph 2034 of "Substances for pharmaceutical use" [22], which is also mandatory and which refers to the general chapter 5.10 about impurities. This general chapter on impurities reflects the principles of the ICH guideline Q3A and gives a decisional flowchart for interpretation.

Last, ICH-Q3B deals with impurities, arising from degradation or interaction, in the finished drug products (FDP). While the settings of specifications was, and still can be, a challenge and often a matter of debate, there has been an evolution towards a consensus around the above mentioned ICH guidelines. In principle, the API synthesis impurities which cannot be degradants in the FDPs are sensu stricto not required to be incorporated in the FDP specifications as they are verified at the API level. However, for transparent consistency and analytical interpretation reasons, the FDP may encompass all related impurities, i.e. not only the degradants and interaction products, but also the API-synthesis impurities. Moreover, independent, external inspections will in practice also consider all related impurities. This principle of a complete impurity profiling in the FDPs is also followed by the International Pharmacopoeia.

As a typical example, the $\mathrm{Ph}$. Eur. monograph on buserelin [21] defines 5 specified impurities, which are thus controlled by the monograph and limited as well. No other detectable impurities (ODIs) are given in the transparency section at the end of the monograph. The general acceptance criterion defined by the specification limit of 'any other impurities', is 3\%, i.e.more than the applicable identification threshold of $0.5 \%$ (Table 2034-2 of monograph 2034), while the disregard limit is $0.1 \%$, identical to the reporting threshold as defined in the general monograph 2034. Following the explanatory chapter 5.10, this means that each of the specified impurities $\mathrm{A}, \mathrm{B}$ and $\mathrm{C}$ are limited to $3 \%$, while impurities D and $\mathrm{E}$ combined are limited to $3 \%$. For the unspecified impurities of buserelin, i.e. HPLC peaks not attributable to impurities $\mathrm{A}$ to $\mathrm{E}$ and which are above the reporting threshold of $0.1 \%$, a specification limit of $0.5 \%$ must be applied. Last, a total related 
Citation: Van Dorpe S, Verbeken M, Wynendaele E, De Spiegeleer B (2011) Purity Profiling of Peptide Drugs. J Bioanal Biomed S6: 003. doi:10.4172/1948-593X.S6-003

impurity limit of $5 \%$ is applied, serving as a boundary limit, and in consistency with the buserelin assay underlimit of 95.0\% (anhydrous, acetic acid-free substance).

If peptides are not chemically produced, but are prepared by biological procedures like products of recombinant DNA technology or fermentation, then additionally, other pharmacopoeial monographs and $\mathrm{ICH}$ guidelines are to be consulted as well for proper setting impurity specifications.

It is important to note that the above regulations are sensu stricto only applicable in a good manufacturing practice (GMP) environment, where quality by design $(\mathrm{QbD})$ risk assessments are promising scientific tools for further efficient drug development (ICH Q8 to Q11 [23-26]). The strict boundary between GMP and "non-GMP" R\&D environment is expected to disappear in the future. Nevertheless, strictly speaking, in current R\&D environment, especially in the discovery phase, no detailed guidances exist about the quality (e.g. impurity profile) of the test items apart from very general statements in the GLP regulations for formal non-clinical studies [27,28].

\section{Peptide synthesis}

Different technologies exist for the production of peptides, ranging from extraction from natural sources, production by recombinant DNA, synthesis in transgenic animals or plants, chemical synthesis and enzymatic synthesis $[1,2,29]$. Apart from financial considerations, the size of the peptide appoints the most suitable technology. Recombinant DNA is particularly applied for the synthesis of large peptides (e.g. insulin, calcitonin and glucagon), while enzymatic synthesis is confined to very small peptides containing less than 10 amino acid residues (e.g. LVVH-7, VV-hemorphin-7 and glutathione). Chemical synthesis remains the gold standard for the production of peptides ranging from 5 to 50 amino acid residues. Although the synthesis was originally performed in solution, after introduction of the solid-phase synthesis mode by Merrifield, this latter method gained more interest [29,30].

\section{Chemical Synthesis}

\section{Solution}

This method is useful for the synthesis of small peptides consisting of only a few amino acid residues. The major advantages consist of easy isolation and purification of intermediates after each synthesis step, better reaction kinetics and the ability to easily characterize intermediates by physical methods such as mass spectrometry and nuclear magnetic resonance (NMR).The main drawback remains the solubility of the protecting groups in order to perform the coupling reaction. Currently, this peptide synthesis method is only rarely used and replaced by solid-phase synthesis $[29,31]$.

\section{Solid-phase peptide synthesis (SPPS)}

SPPS employs an inert and insoluble resin onto which amino acids are attached as a growing chain. Synthesis starts by attaching the first amino acid onto the resin, followed by step-wise coupling of additional amino acids, involving a series of deprotecting, coupling and washing steps (see Figure 1 for a general scheme). The coupling-deprotection is

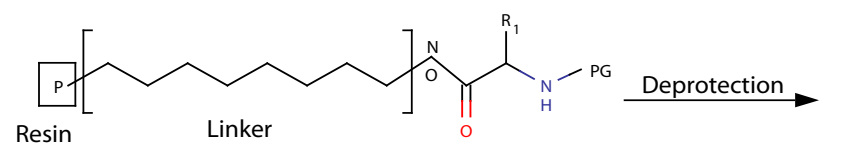

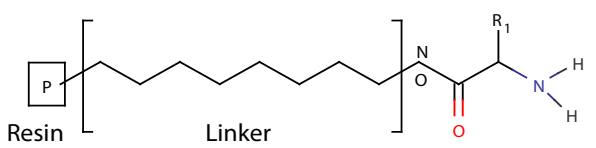
Resin Linker

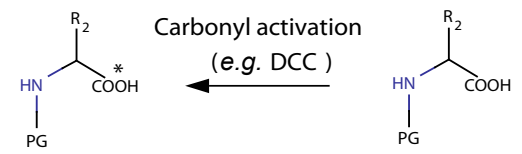<smiles>[2H]C(NC(=O)C(C)(C)CCCCCCCC1CCCC1)C(=O)NC([2H])C1(C)CCCC1</smiles><smiles>NC(N)C(=O)NC([10NH])C(=O)O</smiles>

Figure 1: General solid-phase peptide synthesis scheme. 
Citation: Van Dorpe S, Verbeken M, Wynendaele E, De Spiegeleer B (2011) Purity Profiling of Peptide Drugs. J Bioanal Biomed S6: 003. doi:10.4172/1948-593X.S6-003

repeated until the desired amino acid sequence has been synthesised [29]. Many advantages over the solution method exist: reaction can be automated, solubilisation problem is absent due to linking to the resin, rapid synthesis, excess of reagents are added to drive reaction to completion, and reagents and solvents are easily washed away by filtering the resin $[29,32,33]$. Currently, solid-phase peptide synthesis remains the most reliable pathway. However, compared to synthesis in solution, it may be difficult to adapt the solution phase chemistry to a solid-phase format, the progress of the reaction can be difficult to monitor, peptide purification is only possible after cleaving the peptide from the resin, racemisation can occur during synthesis, side chains of the amino acids need to be protected [29,33]. Several synthesis strategies are developed relating to the choices of the resin, the protecting groups, the coupling reagents and cleavage procedure.

The resin is a solid support consisting of a polymer, which should be chemically inert and stable, mechanically robust, easily filterable and accessible to solvents. Classically, resins are beads of polystyrene (PS) that have been cross linked with divinyl benzene (DVB), but currently, different resins are derivatised with functional groups $[29,33]$. Additionally, a linker may be introduced to attach the first amino acid to the resin and minimise the influence of the resin on the synthesis. The linker should be stable during synthesis and selectively removed to release the product. Acid-sensitive linkers contain an acetal, benzyl or trityl functional group and are cleaved by acids like TFA due to the formation of carbocations. Depending on the linker, different functionalities are incorporated [34]. The standard supports in SPPS are the Merrifield resins, which are chloromethylated PS-DVB linkers to which a wide variety of additional linkers and amino acids can be attached [35]. The Wang resin, consisting of a p-benzyloxybenzylalcohol support, is another very popular SPPS support [36]. Possible benzhydryl linkers are benzhydrylamine(BHA)

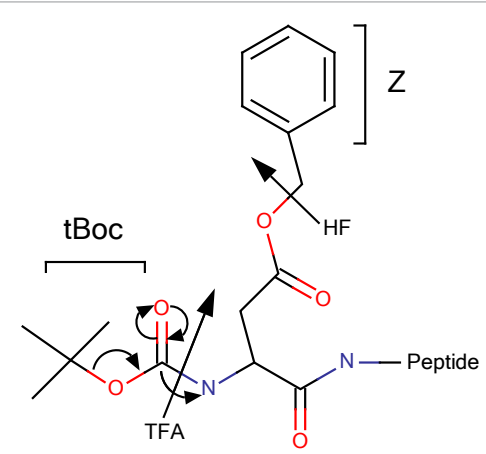<smiles>CC(C)C(=O)OC(C)(C)C(=O)O[Na]</smiles>
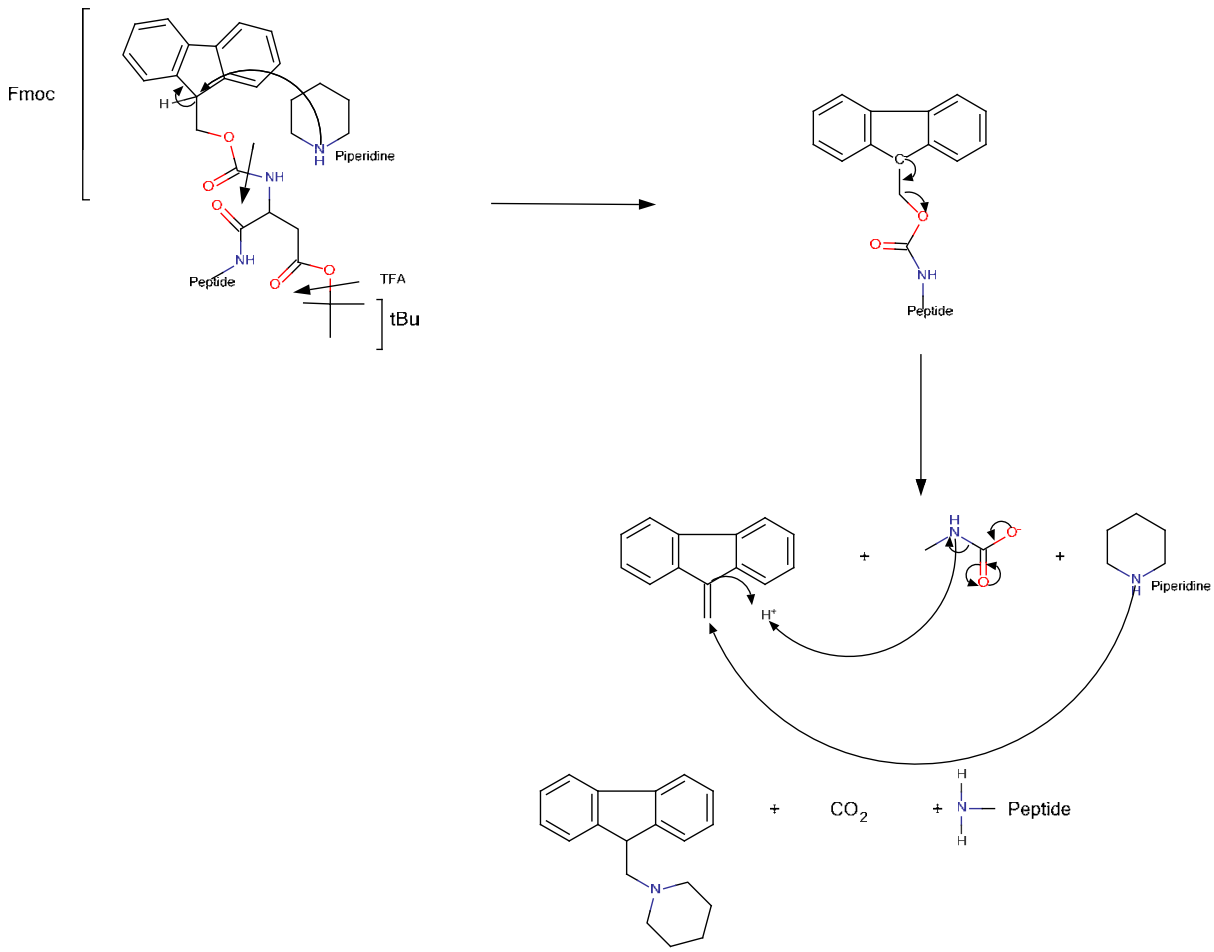

Figure 2: Transient and permanent protecting groups for amino acids: tBoc-Z (a) and Fmoc-tBu (b). 
Citation: Van Dorpe S, Verbeken M, Wynendaele E, De Spiegeleer B (2011) Purity Profiling of Peptide Drugs. J Bioanal Biomed S6: 003. doi:10.4172/1948-593X.S6-003

Page 5 of 18

\begin{tabular}{|c|c|c|c|}
\hline $\begin{array}{l}\text { Amino } \\
\text { Acid }\end{array}$ & Side chain group & tBoc-strategy & Fmoc-strategy \\
\hline Arg & $\mathrm{R}-\mathrm{NH}-\mathrm{C}=\mathrm{NH}-\mathrm{NH}_{2}$ & Tos/Mts/NO $/$ tffa/Z/Alloc & $\begin{array}{l}\text { Tos/Pmc/Pbf/Mts/Mtr/MIS/ } \\
\text { bis-tBoc/Suben/Sub/MeSub/NO } / \text { tffa/Z/Alloc }\end{array}$ \\
\hline Asn & $\mathrm{R}-\mathrm{CONH}_{2}$ & Trt/Xan & Trt/Xan/Mtt/Cpd/Mbh/Tmob \\
\hline Asp & $\mathrm{RCOOH}$ & $\begin{array}{l}\text { OBzl/Bn/cHx/Men/Fmoc/Dmab/Al/ } \\
\text { pNB/Dmnb }\end{array}$ & $\begin{array}{l}\mathrm{OtBu} / \mathrm{Bn} / \mathrm{cH} \text { /tBu/Men/Mpe/ } \\
\text { 2-Ph-iPr/TEGBz/TEGBn/Dmab/Al/pNB/ } \\
\text { PTMSE }\end{array}$ \\
\hline Cys & R-SH & $\begin{array}{l}\text { Meb/Mob/Bn/tBu/1-Ada/Fmoc/Dnpe/ } \\
\text { Acm/PhAcm/StBu/Npys/S-Pyr/ } \\
\text { Alloc/oNB/4-picolyl/Nin }\end{array}$ & $\begin{array}{l}\text { Meb/Mob/Trt/Mmt/Tmob/Xan/Pmbf/Bn/tBu/ } \\
\text { 1-Ada/Acm/PhAcm/StBu/oNB/4-picolyl }\end{array}$ \\
\hline GIn & R-CONH & Trt/Xan & Trt/Xan/Mtt/Cpd/Mbh/Tmob \\
\hline Glu & $\mathrm{RCOOH}$ & $\begin{array}{l}\mathrm{Bn} / \mathrm{cH} / \mathrm{Men} / \mathrm{Fmoc} / \mathrm{Dmab} / \mathrm{Al} / \mathrm{pNB} / \\
\mathrm{Dmnb}\end{array}$ & $\begin{array}{l}\text { Bn/cHx/tBu/Men/Mpe/2-Ph-iPr/TEGBz/ } \\
\text { TEGBn/Dmab/Al/pNB/PTMSE }\end{array}$ \\
\hline His & & Tos/Doc/Bom/Fmoc/Dmbz/Dnp & Trt/Mtt/Mmt/tBoc/Bom/Dmbz \\
\hline Lys & $\mathrm{R}-\mathrm{NH}_{2}$ & $\begin{array}{l}\text { Cl-Z/Z/Alloc/oNBS/dNBS/Troc/Dts/ } \\
\text { pNZ/Poc/oNZ/NVOC/NPPOC/ } \\
\text { MNPPOC/HFA/Fmoc/ivDde/tfa/Msc/ } \\
\text { TCP/Phdec/Pydec/Ddiv }\end{array}$ & $\begin{array}{l}\mathrm{Cl} \text {-Z/tBoc/Z/Alloc/oNBS/Troc/pNZ/oNZ/ } \\
\text { NVOC/NPPOC/MNPPOC/Azoc/Mtt/ } \\
\text { ivDde/TCP/Phdec/Pydec }\end{array}$ \\
\hline Met & R-S- $\mathrm{CH}_{3}$ & $\mathrm{tBu} / \mathrm{Acm} / \mathrm{StBu}$ & Trt/Tmob/ Mmt \\
\hline Ser & $\mathrm{R}-\mathrm{OH}$ & $\mathrm{Bn} / \mathrm{cH} / \mathrm{TBDPS} / \mathrm{Dmnb} / \mathrm{Poc}$ & $\mathrm{Bn} / \mathrm{cHx} / \mathrm{tBu} / \mathrm{Trt} / \mathrm{TBDMS} /$ Pseudoprolines/ TBDPS/Dmnb/Poc \\
\hline Thr & $\mathrm{R}-\mathrm{OH}$ & $\mathrm{Bn} / \mathrm{cHx}$ & $\mathrm{Bn} / \mathrm{cHx} / \mathrm{tBu} / \mathrm{Trt} / \mathrm{TBDMS} /$ Pseudoprolines \\
\hline Trp & & For/Hoc/Mts/Alloc & tBoc/Hoc/Mts \\
\hline Tyr & $\mathrm{R}-\mathrm{Ph}-\mathrm{OH}$ & $\mathrm{Bn} / \mathrm{Dcb} / \mathrm{BrBn} / \mathrm{Z} / \mathrm{BrZ} / \mathrm{Pen} / \mathrm{Al} / \mathrm{oNB} / \mathrm{Poc}$ & $\begin{array}{l}\mathrm{Bn} / \mathrm{tBu} / \mathrm{Dcb} / \mathrm{BrBn} / \mathrm{Pen} / \mathrm{Trt} / 2-\mathrm{Cl}-\mathrm{Trt} / \mathrm{TBDMS} / \\
\text { TEGBz/TEGBn/Al/oNB/tBoc-Nmec }\end{array}$ \\
\hline
\end{tabular}

Table 1: Protecting groups for the side chains of different amino acids.

resin and methylbenzhydrylamine (MBHA) resin $[37,38]$. Trityl linkers are also described, which are highly acid-labile supports [39]. The Rink amide resin (4-(2',4'-dimethoxyphenyl-Fmoc-aminomethyl)phenoxymethyl) contains also phenoxymethyl groups [40]. In general, the majority of these linkers are acid-labile and thus the product is cleaved in acid conditions, whereby the operational conditions, influencing the purity as well, vary widely depending upon the resinlinker type.

Next, a wide variety of protecting groups (PG) exists and is being discovered to protect the amino group (transient PG) as well as side chain groups (permanent PG). The two main transient protecting groups are fluorenylmethyloxycarbonyl (Fmoc) and tert-butyloxycarbonyl (tBoc), which can be combined with tert-butyl (tert-Bu) and benzyl, permanent protecting groups respectively (Figure 2). Because these protecting groups are labile under basic or acidic conditions, a pyruvoyl group was synthesised, which is compatible with the acid-labile benzyl protecting group and is easily cleaved without side reaction [41]. In addition, specific protecting groups are being developed: cyclohexyloxycarbonyl (Hoc) protects the indole ring from oxidation and alkylation in Trp-containing peptides [42]. Another protecting group developed to protect tryptophan is 4 - $(\mathrm{N}$-methylamino)butanoyl (Nmbu) [43]. Water-soluble N-protecting groups are being described as well: methylsulfonylethyloxycarbonyl, 2-phosphonioethyloxycarbonyl, 2-(triphenylphosphonio)isopropyloxycarbonyl, 2-(4-pyridyl)ethyloxycarbonyl, 9-(2-sulfo)fluorenyloxycarbonyl and 2-[phenyl(methyl)sulfonio] ethyloxycarbonyl tetrafluoroborate. However, the latter cannot be used for methionine and cysteine [32]. Currently, protecting groups containing selenium are developed, which seems a very promising protection technique [44]. An overview of the protecting groups used for a specific amino acid according to the tBoc versus Fmoc synthesis strategies is presented in Table 1 [45].

In the next synthesis step, in order to couple the different amino acids, the carboxyl group is activated with coupling agents, such as carbodiimides (e.g. dicyclohexylcarbodiimide DCC), phosgene, anhydrides, esters, acylphosphonium salts (BOP and PyBOP) and uranium salts (HBTU, HATU and TBTU) (Figure 3). This way, reactive compounds as acid chlorides, anhydrides, carbonic anhydrides or esters are formed, which facilitate the production of the peptide bond [46-50].

Additives may be added in order to reduce possible epimerisation during peptide bond formation and fasten the coupling reaction. Mostly 1-hydroxy-benzotriazole (HOBt) is added because of its high reactivity, specificity and coupling yield. Other possible additives are 1-hydroxy-7-azabenzotriazole (HOAt), hydroxysuccinimide (HOSu) 
Citation: Van Dorpe S, Verbeken M, Wynendaele E, De Spiegeleer B (2011) Purity Profiling of Peptide Drugs. J Bioanal Biomed S6: 003. doi:10.4172/1948-593X.S6-003

Page 6 of 18

\begin{tabular}{|c|c|c|}
\hline Monograph & Specified impurities & Other detectable impurities \\
\hline \multirow[t]{5}{*}{ Ph. Eur. 7.3-07/2011:1077 ‘Buserelin’ } & [2-D-histidine]buserelin & \multirow[t]{5}{*}{-} \\
\hline & [4-D-serine]buserelin & \\
\hline & Buserelin-(3-9)-peptide & \\
\hline & [5-D-tyrosine]buserelin & \\
\hline & [1-(5-oxo-D-proline)]buserelin & \\
\hline \multirow{7}{*}{$\begin{array}{l}\text { Ph. Eur. 7.3-01/2008:0471 'Calcitonin (salmon)' } \\
\text { USP33 p1747 }\end{array}$} & Acetylcalcitonin (salmon) & \multirow[t]{7}{*}{-} \\
\hline & [9-D-leucine]calcitonin (salmon) & \\
\hline & Des-22-tyrosine-calcitonin (salmon) & \\
\hline & O-acetylated calcitonin (salmon) & \\
\hline & Salmon calcitoninylglycine $\{\mathrm{rDNA}$ only $\}$ & \\
\hline & [1,7-bis(3-sulpho-L-alanine]calcitonin (salmon) \{rDNA only\} & \\
\hline & $\begin{array}{l}\text { [1,7-bis(3-sulpho-L-alanine]calcitoninylglycine (salmon) } \\
\text { \{rDNA only\} }\end{array}$ & \\
\hline \multirow{7}{*}{$\begin{array}{l}\text { Ph. Eur. 7.3-07/2009:0712 ‘Desmopressin’ } \\
\text { USP33 p2075 }\end{array}$} & \multirow{7}{*}{ Oxytocin } & [5-L-aspartic acid]desmopressin \\
\hline & & [4-L-glutamic acid]desmopressin \\
\hline & & [9-glycine]desmopressin \\
\hline & & [8-L-arginine]desmopressin \\
\hline & & $N^{5.4}-[($ acetylamino $)$ methyl]desmopressin \\
\hline & & $N^{4.5}$-[(acetylamino)methyl]desmopressin \\
\hline & & $N^{1.9}, N^{1.9}$-dimethyldesmopressin \\
\hline \multirow{2}{*}{$\begin{array}{l}\text { R0-CEP 2002-058-Rev 00: Lipotec desmopressin } \\
\text { [valid = R1-CEP 2002-058-Rev 02 - BCN Peptides] }\end{array}$} & [6-D-cysteine]desmopressin $(0.5 \%)$ & \multirow[t]{2}{*}{-} \\
\hline & aspartic acid-dehydrated desmopressin $(0.5 \%)$ & \\
\hline \multirow[t]{6}{*}{ Ph. Eur. 7.3-01/2008:1634 'Felypressin’ } & $S^{1}, S^{6}$-bis[(acetylamino)methyl]-(reduced felypressin) & \multirow[t]{6}{*}{-} \\
\hline & [5-aspartic acid]felypressin & \\
\hline & bis(reduced felypressin) (1,6'),(1',6)-bis(disulfide) & \\
\hline & bis(reduced felypressin) $\left(1,1^{\prime}\right),\left(6^{\prime}, 6\right)$-bis(disulfide) & \\
\hline & $N^{1}$-acetylfelypressin & \\
\hline & [4-glutamic acid]felypressin & \\
\hline \multirow{2}{*}{$\begin{array}{l}\text { Ph. Eur. 7.3-01/2008:0827 'Gonadorelin acetate' } \\
\text { USP33 p2519 }\end{array}$} & - & \multirow[t]{2}{*}{-} \\
\hline & Gonadorelin free acid & \\
\hline \multirow{2}{*}{$\begin{array}{l}\text { R0-CEP 2005-022-Rev 00: Bachem gonadorelin } \\
\text { acetate } \\
\text { [currently valid] }\end{array}$} & [2-D-histidine]gonadorelin (0.5\%) & \multirow[t]{2}{*}{-} \\
\hline & [5-D-tyrosine]gonadorelin $(0.5 \%)$ & \\
\hline \multirow[t]{12}{*}{ Ph. Eur. 7.3-01/2008:1636 ‘Goserelin’ } & [4-D-serine]goserelin & \multirow[t]{12}{*}{-} \\
\hline & [6-[O-(1,1-dimethylethyl)-L-serine]goserelin & \\
\hline & [9-D-proline]goserelin & \\
\hline & $\begin{array}{l}\text { 1-carbomoylyl-2-[5-oxo-L-prolyl-L-histidyl-L-tryptophyl-L- } \\
\text { seryl-L-tyrosyl-O-(1,1-dimethylethyl)-D-seryl-L-leucyl-L- } \\
\text { arginyl]diazane }\end{array}$ & \\
\hline & $\begin{array}{l}\text { 5-oxo-L-prolyl-L-histidyl-L-tryptophyl-L-seryl-L-tyrosyl- } \\
\text { O-(1,1-dimethylethyl)-D-seryl-L-leucyl-L-arginyl-L- } \\
\text { prolinohydrazide }\end{array}$ & \\
\hline & [5-D-tyrosine]goserelin & \\
\hline & [2-D-histidine]goserelin & \\
\hline & [1-(5-oxo-D-proline)]goserelin & \\
\hline & endo-8a,8b-di-L-proline-goserelin & \\
\hline & endo-8a-L-proline-goserelin & \\
\hline & $\mathrm{O}^{4}$-acetylgoserelin & \\
\hline & [7-D-leucine]goserelin & \\
\hline \multirow{5}{*}{$\begin{array}{l}\text { Ph. Eur. 7.3-01/2008:0907 'Gramicidin' } \\
\text { USP33 p2528 }\end{array}$} & [4-methionine]gramicidin A1 & \multirow[t]{5}{*}{-} \\
\hline & Gramicidin A1 3-hydroxypropyl & \\
\hline & Gramicidin B2 & \\
\hline & [10-methionine]gramicidin C1 & \\
\hline & Gramicidin A2 3-hydroxypropyl & \\
\hline $\begin{array}{l}\text { Ph. Eur. 7.3-01/2008:1637 'Bovine insulin' } \\
\text { USP33 p2639 }\end{array}$ & A-21 desamido insulin & - \\
\hline
\end{tabular}


Citation: Van Dorpe S, Verbeken M, Wynendaele E, De Spiegeleer B (2011) Purity Profiling of Peptide Drugs. J Bioanal Biomed S6: 003. doi:10.4172/1948-593X.S6-003

Page 7 of 18

\begin{tabular}{|c|c|c|}
\hline \multirow{3}{*}{$\begin{array}{l}\text { Ph. Eur. 7.3-01/2011:0838 ‘Human insulin' } \\
\text { USP33 p2642 } \\
\text { IP2010 }\end{array}$} & - & \multirow[t]{3}{*}{-} \\
\hline & A-21 desamido insulin & \\
\hline & - & \\
\hline \multirow{2}{*}{$\begin{array}{l}\text { Ph. Eur. 7.3-01/2008:2085 ‘Insulin lispro’ } \\
\text { USP33 p2644 }\end{array}$} & - & \multirow[t]{2}{*}{-} \\
\hline & A-21 desamido insulin lispro & \\
\hline Ph. Eur. 7.3-01/2008:2084 'Insulin aspart' & - & - \\
\hline $\begin{array}{l}\text { Ph. Eur. 7.3-01/2008:1638 'Insulin porcine' } \\
\text { USP33 p2639 }\end{array}$ & A-21 desamido insulin & - \\
\hline Ph. Eur. 7.3-07/2010:2215 ‘lodixanol’ USP33 p2660 & - & - \\
\hline \multirow{7}{*}{$\begin{array}{l}\text { Ph. Eur. 7.3-01/2008:1442 ‘Leuprorelin’ } \\
\text { USP33 'Leuprolide' p2761 }\end{array}$} & [4-D-serine]leuprorelin & [3-D-tryptophane]leuprorelin \\
\hline & [2-D-histidine]leuprorelin & [2-D-histidine,4-D-serline]leuprorelin \\
\hline & [6-L-leucine]leuprorelin & [5-D-tyrosine]leuprorelin \\
\hline & [4-(O-acetyl-L-serine)]leuprorelin & [7-D-leucine]leuprorelin \\
\hline & & [1-(5-oxo-D-proline]leuprorelin \\
\hline & & $\begin{array}{l}\text { [8-[5- } N \text {-[imino(1H-pyrazol-1-yl)methyl]-L-ornithine]] } \\
\text { leuprorelin }\end{array}$ \\
\hline & & [4-dehydroalanine]leuprorelin \\
\hline \multirow{3}{*}{$\begin{array}{l}\text { R0-CEP 2001-454-Rev 01: Bachem leuprorelin } \\
\text { [valid = R1-CEP 2001-454-Rev 01] }\end{array}$} & Pyr-His-Trp-Ser-Tyr-OH (0.5\%) & \\
\hline & des-Pro-NHEt'-leuprorelin $(0.2 \%)$ & \\
\hline & unidentified impurity at RRT $=0.87(0.2 \%)$ & \\
\hline \multirow{3}{*}{$\begin{array}{l}\text { Ph. Eur. 7.3-01/2008:0780 ‘Oxytocin’ } \\
\text { USP33 p3185 } \\
\text { Ph. Int. }\end{array}$} & - & \multirow[t]{6}{*}{-} \\
\hline & - & \\
\hline & Carbimido oxytocin & \\
\hline \multirow[t]{3}{*}{ IP2010 } & Acetyloxytocin & \\
\hline & $\begin{array}{l}\text { A-oxytocin dimer } \\
\text { B-oxytocin dimer }\end{array}$ & \\
\hline & - & \\
\hline \multirow[t]{5}{*}{ Ph. Eur. 7.3-01/2008:1144 'Protirelin' } & 5-oxo-L-prolyl-D-histidyl-L-prolinamide & \multirow[t]{5}{*}{-} \\
\hline & 5-oxo-D-prolyl-L-histidyl-L-prolinamide & \\
\hline & 5-oxo-L-prolyl-L-histidine & \\
\hline & 5-oxo-L-prolyl-L-histidyl-L-proline & \\
\hline & cyclo(-L-histidyl-L-prolyl-) & \\
\hline Ph. Eur. 7.3-01/2011:0949 ‘Somatostatin’ & - & - \\
\hline \multirow{11}{*}{$\begin{array}{l}\text { R0-CEP 2005-245-Rev 00: Bachem somatostatin } \\
\text { [currently valid] }\end{array}$} & unidentified impurity at RRT $=0.68-0.72(0.5 \%)$ & \multirow[t]{11}{*}{-} \\
\hline & unidentified impurity at RRT $=0.77(0.5 \%)$ & \\
\hline & unidentified impurity at RRT $=0.80(0.5 \%)$ & \\
\hline & Somatostatin-(1-14)-peptide (0.5\%) & \\
\hline & unidentified impurity at RRT $=0.92(0.5 \%)$ & \\
\hline & [12-(O-acetyl-threonine)]somatostatin $(0.5 \%)$ & \\
\hline & [13-(O-acetyl-L-serine)]somatostatin $(0.8 \%)$ & \\
\hline & unidentified impurity at RRT $=1.31(0.8 \%)$ & \\
\hline & $(N-\varepsilon$-acetyl-lysine)somatostatin $(0.5 \%)$ & \\
\hline & unidentified impurity at RRT = $1.43(0.5 \%)$ & \\
\hline & unidentified impurity at RRT $=1.65-1.74(0.5 \%)$ & \\
\hline \multirow[t]{2}{*}{ Ph. Eur. 7.3-04/2010:0644 'Tetracosactide’ } & tetracosactide sulphoxide & \\
\hline & unidentified impurity at RRT $=0.95$ & \\
\hline \multirow{3}{*}{$\begin{array}{l}\text { Ph. Eur. 7.3-01/2011:0580 'Aprotinin' } \\
\text { USP33 p1575 }\end{array}$} & Aprotinin-(1-56)-peptide & \\
\hline & Aprotinin-(1-57)-peptide & \\
\hline & Pyroglutamyl aprotinin & \\
\hline $\begin{array}{l}\text { Ph. Eur. 7.3-01/2008:1635‘Human glucagon’ } \\
\text { USP33 p2504 }\end{array}$ & - & - \\
\hline \multirow{2}{*}{$\begin{array}{l}\text { Ph. Eur. 7.3-01/2008:0465‘Bacitracin’ USP33 p1620 } \\
\text { IP } 2010\end{array}$} & Bacitracin F & \\
\hline & Impurity E & \\
\hline \multirow{3}{*}{$\begin{array}{l}\text { IP2010: Bleomycin } \\
\text { USP33 p1693 }\end{array}$} & Demethylbleomycin A2 & \\
\hline & Bleomycinic acid & \\
\hline & Bleomycin B4 & \\
\hline
\end{tabular}


Citation: Van Dorpe S, Verbeken M, Wynendaele E, De Spiegeleer B (2011) Purity Profiling of Peptide Drugs. J Bioanal Biomed S6: 003. doi:10.4172/1948-593X.S6-003

Page 8 of 18

\begin{tabular}{|l|l|}
\hline USP33: 'Capreomycin' p1777 IP2010 & - \\
\hline IP2010:' Cephalexin' & D-phenylglycine \\
\hline USP33 p1870 & 7- aminodesacetoxycephalosporanic acid \\
\hline USP33: 'Dactinomycin' p2053 & cefotaxime \\
\hline IP2010: Vasopressin & - \\
\hline USP33 p3849 & Oxytocin \\
\hline $\begin{array}{l}\text { IP2010: Vancomycin } \\
\text { USP33 p3844 }\end{array}$ & N-demethylvancomycin B \\
\hline & aglucovancomycin B \\
\hline & $\beta$-Asp3 vancomycin B \\
\hline & desvancosaminylvancomycin B \\
\hline
\end{tabular}

Table 2: Pharmacopoeial peptides.

or N-hydroxy-5-norbornene-2,3-dicarboximide (HONB) [32,51]. An overview of the structures of the frequently applied scavengers is presented in Figure 4.

Finally, the peptide is cleaved from the resin after completing the peptide sequence. In this step, scavengers like p-cresol, anisole and Fmoc-Leu are added to reduce the unwanted side reactions of the reactive species like carbocations formed during the acid cleavage. However, these scavengers may also lead to the unwanted formation

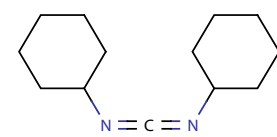

DCC<smiles>O=C1CCC(=O)N1O</smiles>

HOSu<smiles>C/N=C(\N=C(\C)n1n[n+]([O-])c2ccccc21)C(F)(F)F</smiles>

HBTU<smiles>CN=C(N)N1N=[N+]([O-])C2CCCCC21</smiles>

TBTU

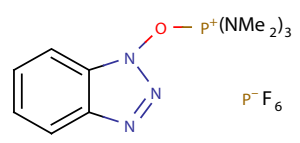

BOP
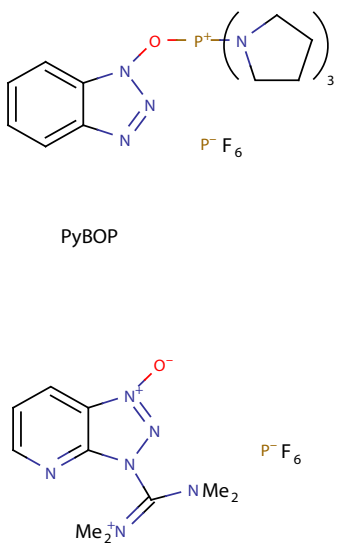

HATU<smiles>On1nnc2cccnc21</smiles>

HOAt
Figure 3: Typical agents used for amino acid coupling.<smiles>COc1ccccc1</smiles>

(thio)arisol

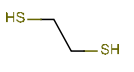

1.2-ethanedithiol

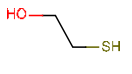

2-mercaptoethano

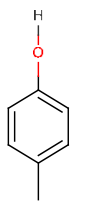

p-cresol<smiles>Cc1cccc2[nH]ccc12</smiles><smiles>Sc1ccccc1</smiles>

thiophenol<smiles>C1CCNCC1</smiles>

piperidine

Figure 4: Structures of typical scavengers. of product adducts $[10,42]$. When producing highly hydrophobic peptides, dissolving problems may occur after cleaving the peptide from the resin. Solubility can be improved by adding charged residues as arginine or by modifying the phenolic alcohol of tyrosine or the $\mathrm{N}$-(2-hydroxy-4-methoxybenzyl) (Hmb) protective group with tBoc$\mathrm{N}$-methyl-N-[(2-methylamino)ethylcarbamoyl (tBoc-Nmec). This way, the Nmec group stays attached to the peptide during cleaving and is removed under basic conditions [43]. As large peptides tend to fold and adopt a unique three-dimensional structure, synthesis may be hampered as well. In order to circumvent this problem, the depsipeptide or $\mathrm{O}$-acyl isopeptide method was reported. Here, the depsipeptide analogue is attached to the resin instead of the peptide, replacing the peptide bond by an ester bond. After completing the whole sequence, the $\mathrm{O}$-acyl isomer is converted into the target peptide. This way, folding is largely reduced and synthesis is facilitated [52,53].

Chemical ligation is an approach for coupling peptide fragments to form the desired peptide sequence and is more interesting for longer peptide sequences. This methodology is frequently applied for the synthesis of cyclic peptides using the intramolecular ligation reaction $[54,55]$. 
Citation: Van Dorpe S, Verbeken M, Wynendaele E, De Spiegeleer B (2011) Purity Profiling of Peptide Drugs. J Bioanal Biomed S6: 003. doi:10.4172/1948-593X.S6-003

\section{Microwave synthesis}

Alternatively to conventional heating, microwave radiation can be applied to introduce energy into the peptide synthesis reaction, resulting in acceleration of the reaction. The products are dissolved in solvents, which convert electromagnetic energy into heat energy due to dielectric polarisation. The claimed major advantages of this technique are the reduced reaction time and the increased synthesis yield as well as purity. However, there is a lack of controllability and reproducibility. Moreover, it is generally believed that undesirable side reactions would also be accelerated by microwave heating and that some coupling reagents are heat sensitive. Nevertheless, SPPS with microwave was successfully applied in the synthesis of peptides [56-58].

\section{Enzymatic synthesis}

Enzymes are biological catalysts, being active at mild conditions ( $\mathrm{pH} 6$ to 8 ), robust and relatively stable (e.g. when using immobilized enzymes), stereo-and regioselective and not requiring stoichiometric cofactors. The proteases mostly used for peptide synthesis are selected based on their specificity towards amino acid residues on each side of the splitting point. The major drawbacks of this method compared to the chemical solution or solid-phase methods consists of the lower productivity, high cost of biocatalysts, broad specificity of the proteases, use of non-conventional reaction media and limited validation and scale-up protocols exist [29]. Moreover, during peptide synthesis, the growing peptide can be simultaneously attacked by the proteases. The use of non-aqueous solvents is also limited due to the low activity of most enzymes in those solvents. Before starting the synthesis, most operational parameters, such as $\mathrm{pH}$, temperature and organic solvent concentration should be optimized, making generic automated synthesis almost impossible. Thus, enzymatic synthesis is dependent on the size of the peptide, being restricted to small hydrophilic peptides. The main advantage of this method is the reduced need to protect the side chains due to the specificity of the reaction $[29,59]$. Because stoichiometric amounts of coupling reagents are not required and racemization does not occur, purification is much easier [60]. Enzymes can also be used in the direct coupling of peptide fragments [61].

\section{Recombinant DNA synthesis}

The recombinant DNA technology involves insertion of the desired protein gene into an expression vector, which contains a promoter to express the protein. After synthesis, the recombinant peptide material is purified by affinity chromatography. Although, a large scale production is possible, the main drawback of this method consists of the long and expensive development procedure. Human insulin is prepared with this method by inserting the insulin gene into the E. Coli bacterial cell. This way, the implanted DNA induces the cell to produce insulin $[62,63]$.

\section{Purity of peptides}

The importance of performant quality control before starting (biomedical) experiments in order to avoid false negative or positive results have been reported previously $[5,10]$. Only selective, reproducible, sensitive techniques can be used to determine and

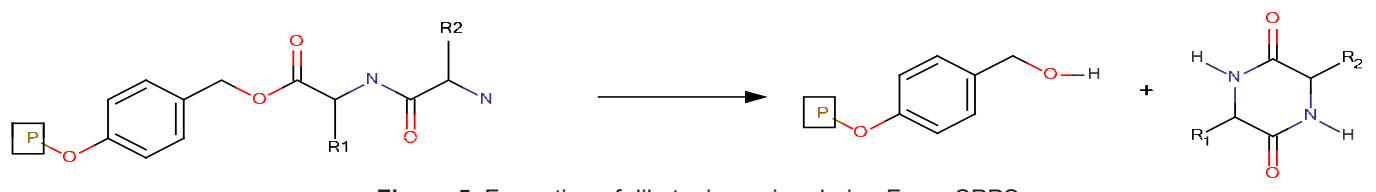

Figure 5: Formation of diketopiperazine during Fmoc-SPPS.

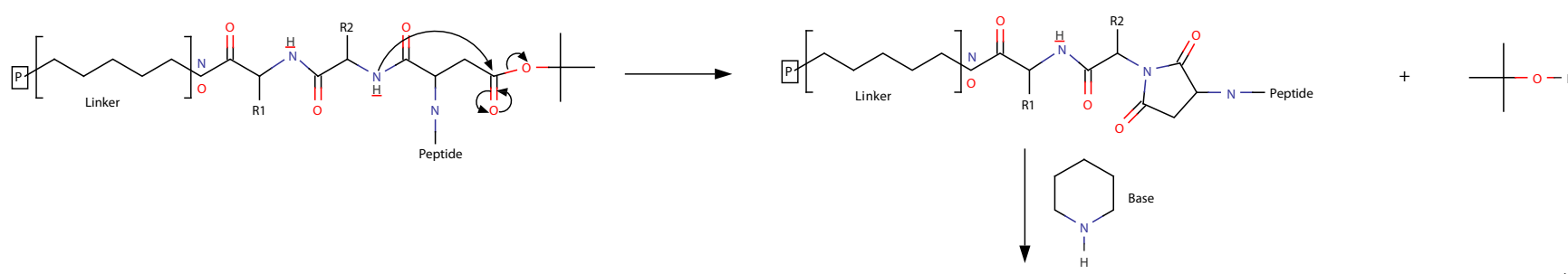

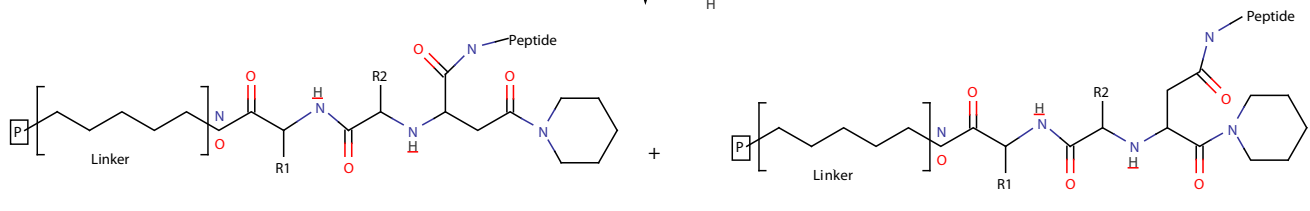

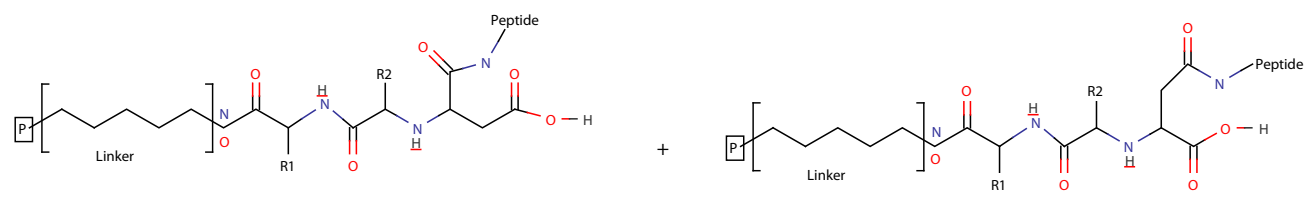

Figure 6: Aspartimide formation in Asp-Gly, Asp-Ala or Asp-Ser sequences 
Citation: Van Dorpe S, Verbeken M, Wynendaele E, De Spiegeleer B (2011) Purity Profiling of Peptide Drugs. J Bioanal Biomed S6: 003. doi:10.4172/1948-593X.S6-003

Page 10 of 18

quantify the purity of the peptide. Traditionally, reversed-phase HPLC with UV detection (RP-HPLC-UV) is applied due to its selectivity, the low limit of detection and quantitation, robustness and high sensitivity, while the HPLC-MS coupling allows the identification of the possible impurities [64,65]. Alternative separation techniques to HPLC may include normal-phase HPLC, thin layer chromatography (TLC) and capillary zone electrophoresis (CZE), while other detection techniques may consist of NMR, fluorescence, refractive index and evaporative light scattering [66,67].

As stated in the section above, during synthesis, different side-chain protecting groups, activating functional groups or scavengers may be added to prevent undesired side-chain reactions. Since chemical synthesis reactions are commonly incomplete, not yielding 100\%, these groups or part(s) of it may remain present or attached to the peptide leading to the presence of closely related peptide impurities. In Table 2, an overview of the typical peptide impurities currently described in the Ph. Eur., USP, IP and Ph. Int. is given $[13,68,69]$.

Next to incomplete removal of the protecting group (tBoc or Fmoc) [68], the repetitive TFA treatments represent sources of oxidation and alkylation side-reactions for the most sensitive amino acids, such as Cys, Met and Trp.
Alkylation by carbocations, generated in the acidolytic cleavage step, is the most prominent side-reaction, but a rational choice of scavengers will help to minimize unwanted modifications. The extent of diketopiperazine formation (Figure 5) can be reduced because the $\mathrm{N}$-amino function is protonated and less prone to attack the benzyl ester bond after the deprotection reaction in tBoc SPPS compared to the situation in Fmoc based SPPS [70]. Another side reaction consists of the aspartimide formation in peptides containing the Asp-Gly, Asp-Ala or Asp-Ser sequences. This reaction is a result of ring closure between the carboxy side chain of Asp and the nitrogen of the carboxamide. The produced aspartimides are very susceptible to base-catalyzed epimerization and undergo rapid ring-opening reactions, yielding mixtures of aspartyl peptides and piperidides as illustrated in Figure 6 [71-73]. In addition, the C-terminal Met can cyclize to homoserine lactone during the HF cleavage if tert-butyl type protecting groups have not been cleaved before the HF treatment as shown in Figure 7 [74]. N-O shift or O-isoacyl peptide formation (Figure 8) can occur during the treatment with strong acid of peptides containing Ser or Thr, but the reaction can be reversed by base treatment $[75,76]$. This intramolecular reaction causes a disruption in the secondary structure of the peptide, increasing solubility [77]. The $\gamma$-carboxyl function of Glu, protonated<smiles>CCCCCCCCCC(NC(CCSC)C(=O)OCc1cccc(CC)c1)C(C)(C)Cl</smiles><smiles>CCCCNC(CCS(C)(C)C(C)(C)C)C(=O)OCc1cccc(C(C)C)c1</smiles>

Cleavage
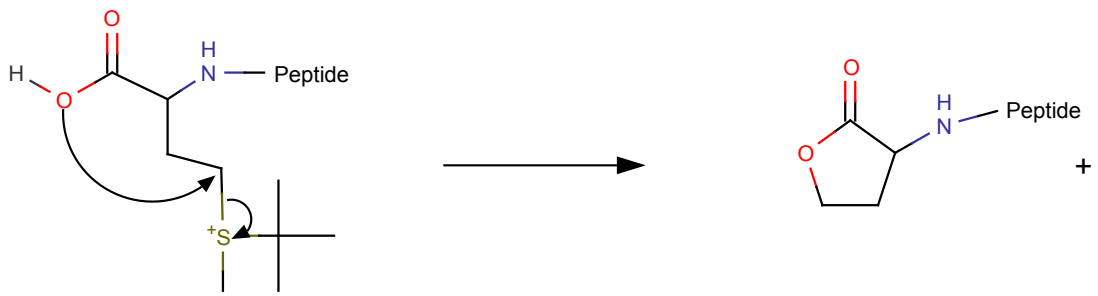

Figure 7: Cyclization of Met to homoserine lactone. 
Citation: Van Dorpe S, Verbeken M, Wynendaele E, De Spiegeleer B (2011) Purity Profiling of Peptide Drugs. J Bioanal Biomed S6: 003. doi:10.4172/1948-593X.S6-003
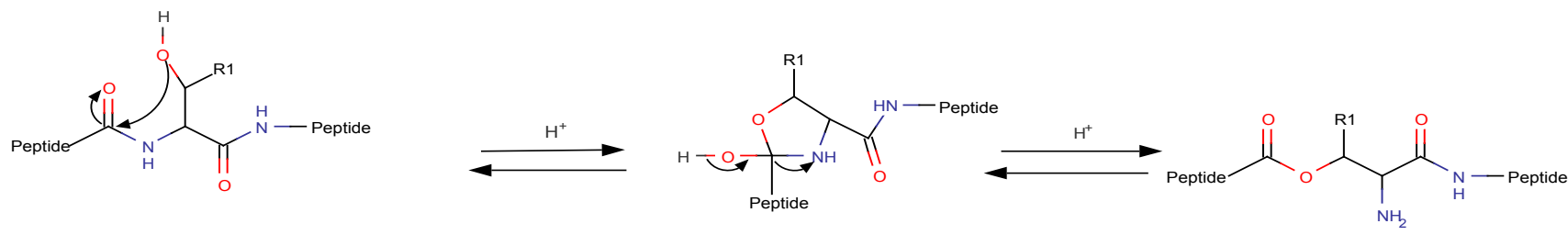

Figure 8: NO-shift during acid treatment.
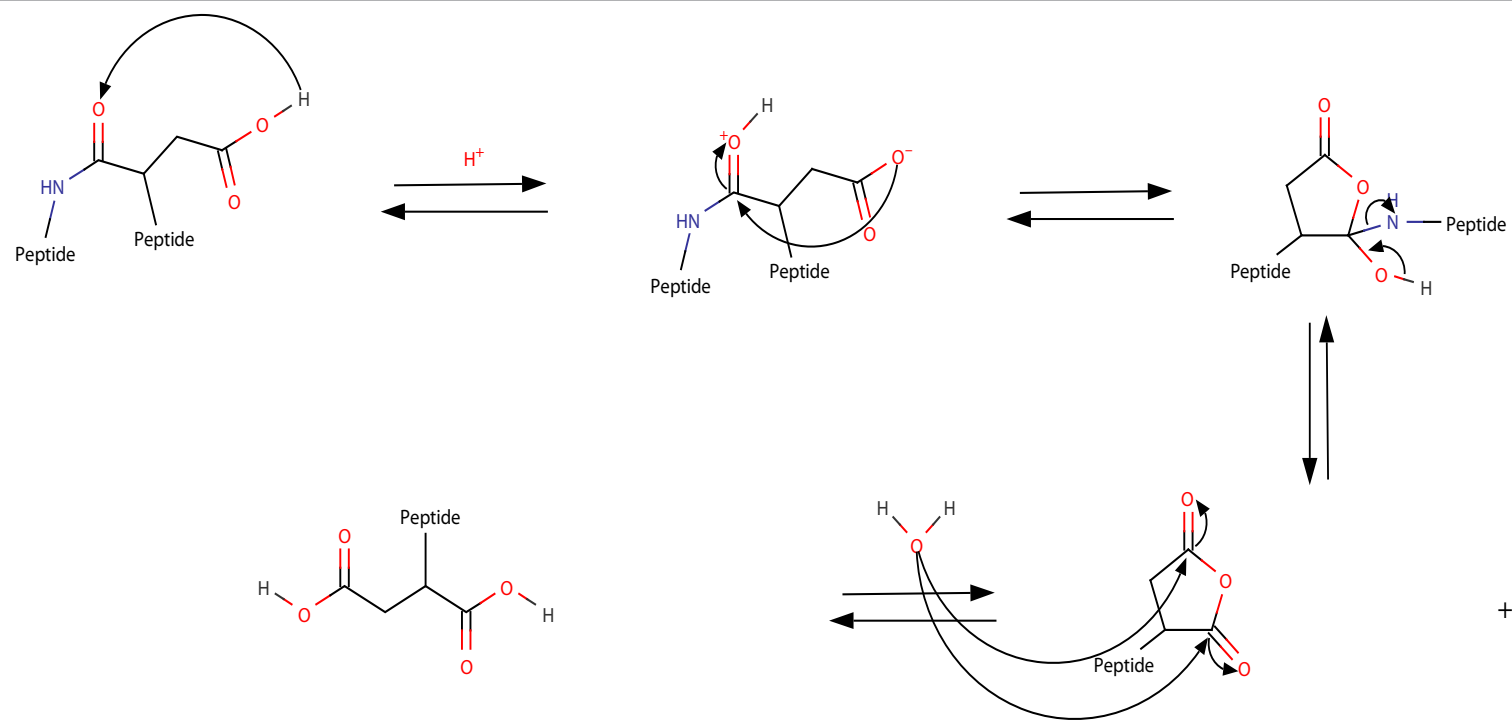

Figure 9: Asp-X bond cleavage.

in strong acid, can lose water and form an acylium ion which will cyclize to a pyrrolidone or be trapped by a scavenger such as anisole to yield a ketone [78]. The cleavage of the Asp-Pro and Asp-Trp bond (Figure 9 ) has been observed during HF treatment $[68,79]$. The adjacent amino acid affects the hydrolytic rate of this cleavage reaction, with Asp-Pro being the fastest [80]. Oxidation of Cys, Met or Trp may occur during cleavage from the resin in $\mathrm{tBoc}$ and Fmoc chemistry, creating a $16 \mathrm{amu}$ mass shift [68]. Serine is protected with tert-butyl, corresponding with a $\mathrm{m} / \mathrm{z}$ value of 56 . A trityl protecting group is introduced for prevention of His, Asn or Gln, shifting the mass with $243 \mathrm{amu}$. When applying the Fmoc chemistry, the 2,2,5,7,8-pentamethylchroman-6-sulfonyl (Pmc) group may not be completely removed from Arg or it may alkylate Trp, creating an impurity with a mass shift of 266 amu. Formylation may occur during tBoc-deprotection causing a mass shift of $28 \mathrm{amu}$, while during Fmoc-synthesis deletions were observed for Asp. For this amino acid, acid- as well as base-catalyzed aspartimide formation is observed, even when protecting with $\beta$-1-adamantyl (1-Ada) group or tert-butyl. In order to reduce this side-reaction when using bases, a new protecting group was developed: $\beta$-3-methylpent3-yl ester [81].

During the protection of Val using Fmoc-oxysuccinimide(FmocOsu), Fmoc- $\beta$-Ala was detected as a major impurity. This underestimated contamination is avoided when replacing Fmoc-Osu by $\mathrm{Fmoc}-\mathrm{Cl}$ [82]. In addition, dimers may be formed by a disulfide linkage [68]. Impurities having the same molecular weight as the drug substance itself represent diastereoisomers [65]. In some syntheses, it was even observed that the wrong amino acid was incorporated in the peptide sequence [83].

In order to prevent possible side-reactions, scavengers may be added. Typical examples are: (thio)anisole, dimethyl sulfide, p-cresol, 1,2-ethanedithiol, 4-methylindole, thiophenol, 2-mercaptoethanol, piperidine or ethylmethylsulfide [68,84]. Anisole is acting as a carbocation scavenger, which are created by strong acid cleavage from the resin. These carbocations present possible side-reactions as they are alkylating species of Cys, Met, Trp and Tyr, containing a nucleophilic center [84]. Typically, anisole or p-cresol react with the acylium ion formed from the dehydration of the side chains of Asp in strong acidic medium, thereby yielding stable ketone impurities [84]. Although these scavengers may reduce the occurrence of possible side-chain reactions, they may not be completely removed and remain present as an impurity.

Dysfunction of deprotecting Trp was reported to leave an anisyl group attached to Trp, leading to a mass shift of $107 \mathrm{amu}$. In addition, impurities may be introduced by the solvents used. Although highly pure solvents (HPLC grade) are used, they may still contain some impurities at low levels, inducing unwanted reactions [67]. In Figure 10, some typical examples of impurities observed in synthesized peptides are presented. The overview of the possible impurities stipulates the importance to investigate peptide impurity before applying to (biomedical) analyses in order to prevent false negative/positive conclusions. One should be aware that these impurities may induce 


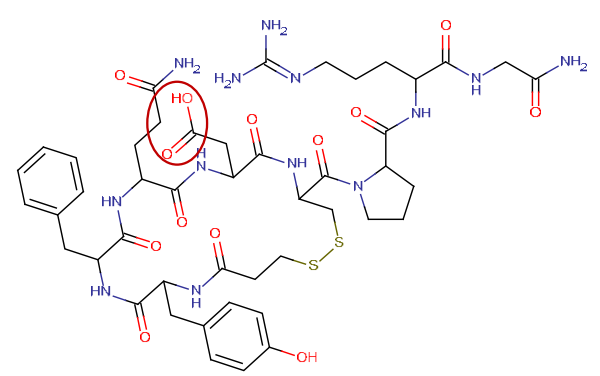

Desamidation of Asp

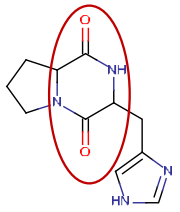

Cyclisation

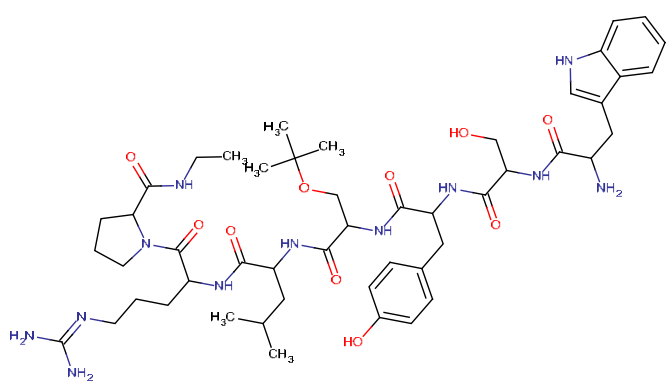

Truncated

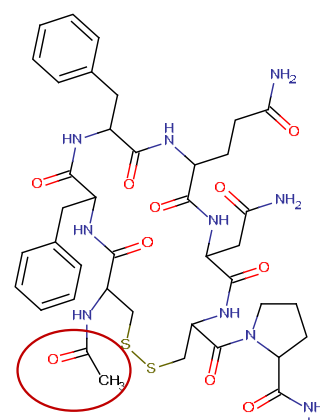<smiles>CCCCCC(NC=O)C(=O)NCC(=O)C(C)(C)C(=O)O</smiles>

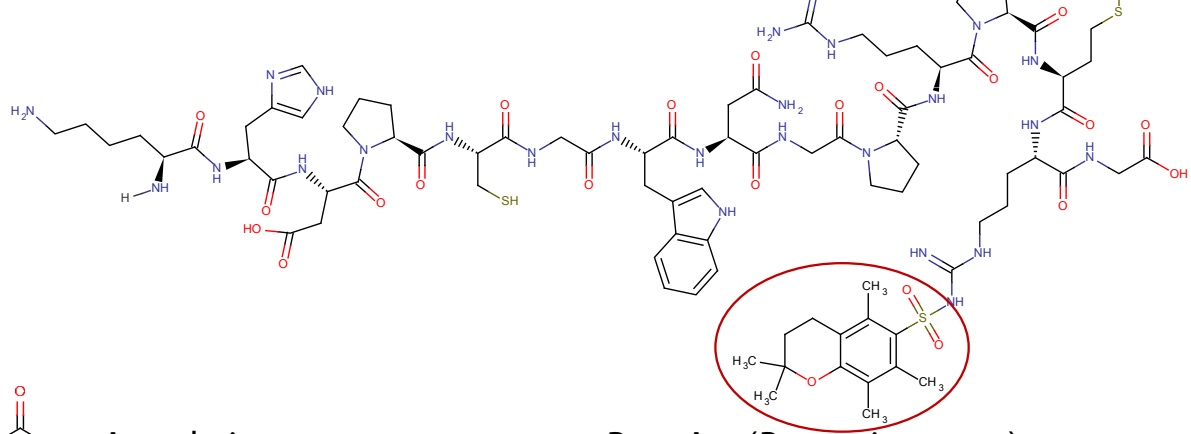

Pmc-Arg (Protecting group)

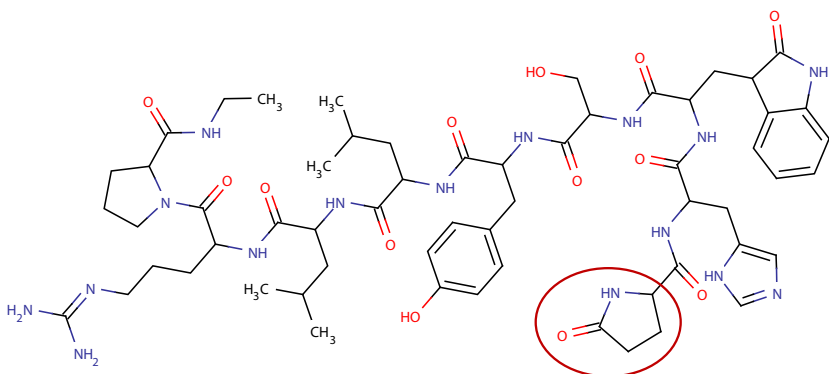

Oxidation

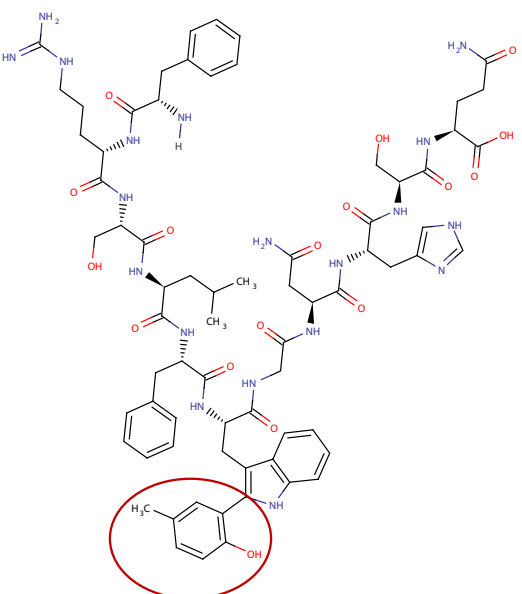

p-Cresol adduct

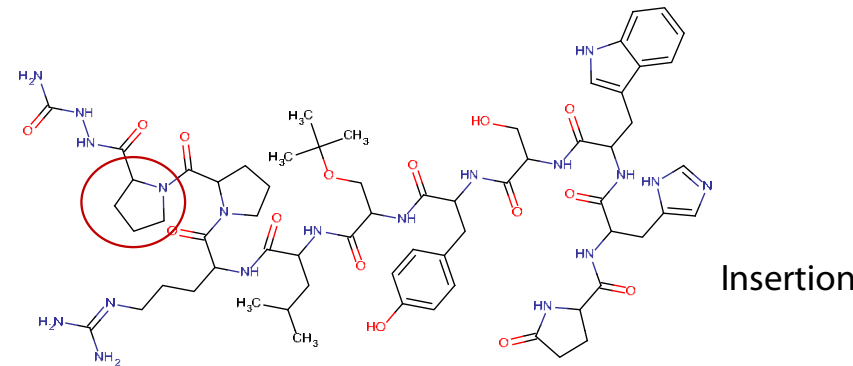

Figure 10: Typical examples of impurities observed in synthesized peptides: desamidation of Asp in desmopressin; 3-9 truncated buserelin; acetyl felypressin; cyclic protirelin; incomplete removal of p-cresol scavenger in SBO215-7; incomplete removal of protecting group Pmc on Arg; insertion of proline in goserelin and oxidized Trp in leuprorelin. 
Citation: Van Dorpe S, Verbeken M, Wynendaele E, De Spiegeleer B (2011) Purity Profiling of Peptide Drugs. J Bioanal Biomed S6: 003. doi:10.4172/1948-593X.S6-003

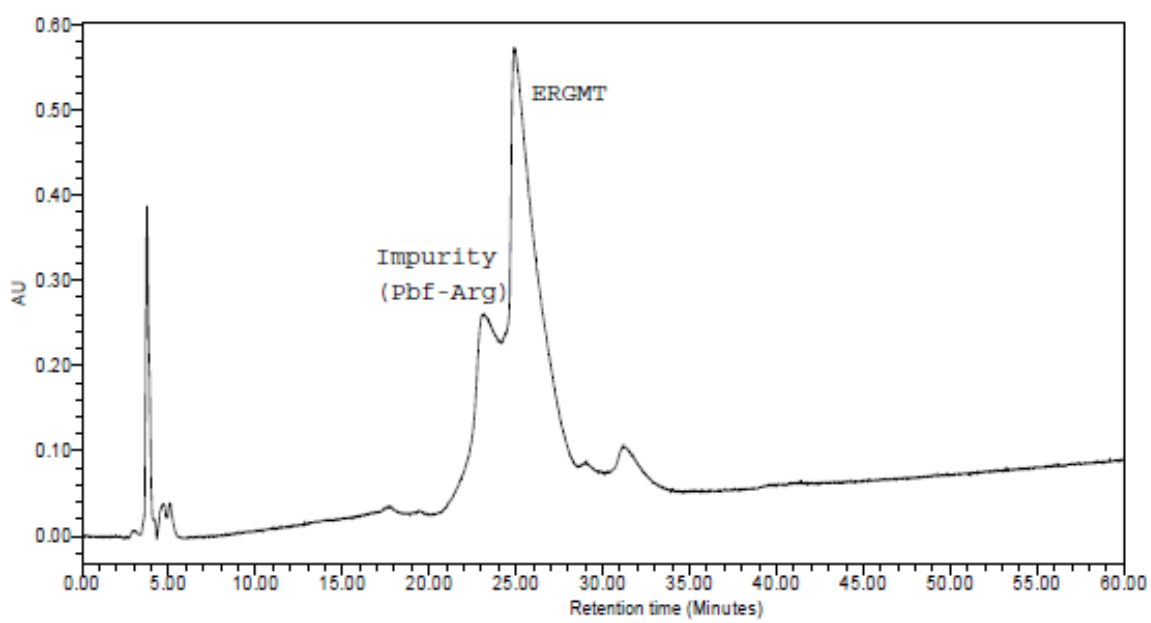

Figure 11: Chromatogram of the impure quorum-sensing peptide "competence and sporulation factor" with sequence ERGMT. The Pbf protecting group of Arg was not completely removed.<smiles>CCCCCCCCC(=O)NCC(N)=O</smiles>

Asn

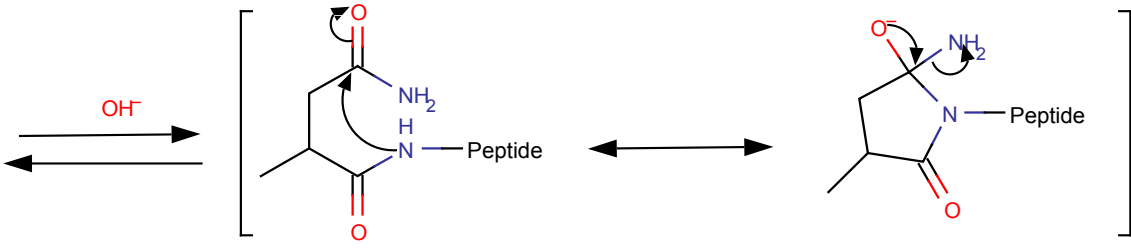

$\mathrm{H}^{+} \mid$<smiles>CCCCCCCC(=O)NCC(=O)O</smiles>

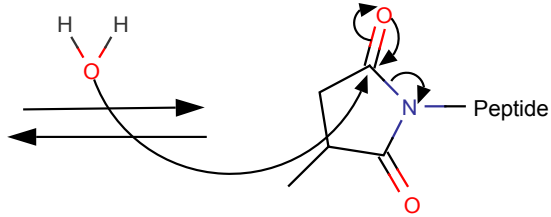

$+\mathrm{NH}_{3}$

iso Asp

Asp

Figure 12: Deamidation of Asn to Asp.

or alter activity of the compound confounding the conclusions $[10,85-$ 88]. Figure 11 shows the chromatogram of an impure quorum sensing peptide, in which the protecting group of Arg was not completely removed.

\section{Degradation of peptides}

Chemical degradation is residue specific and thus controlled by the primary peptide sequence [89]. For example, among the amino acids, Asn and Asp are the most unstable residues [90].The major chemical degradation reactions are: deamidation, hydrolysis, peptide bond cleavage, oxidation, Maillard reaction, $\beta$-elimination, enantiomerization, isomerization and dimerization [67,90-92]. Next to stability issues of the peptide APIs or drug substance, formulated finished drug products (FDPs) show also degradation. A formulated FDP comprises only one, or a limited number and quatity, drug substance and multiple excipients, which are included i.a. to prevent degradation during processing and/or storage. Depending on the formulation, different stability patterns are observed. Generally, a dry formulation is more stable than an aqueous formulation. However, these excipients or impurities that are present in the excipients may also react with the peptides, inducing degradation $[67,91,93,94]$. Thus, it is crucial to select the most appropriate excipient $[95,96]$. 
Citation: Van Dorpe S, Verbeken M, Wynendaele E, De Spiegeleer B (2011) Purity Profiling of Peptide Drugs. J Bioanal Biomed S6: 003. doi:10.4172/1948-593X.S6-003

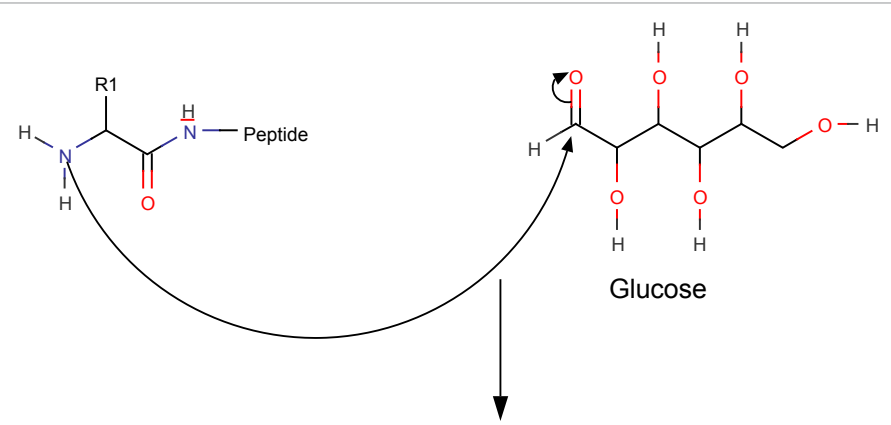

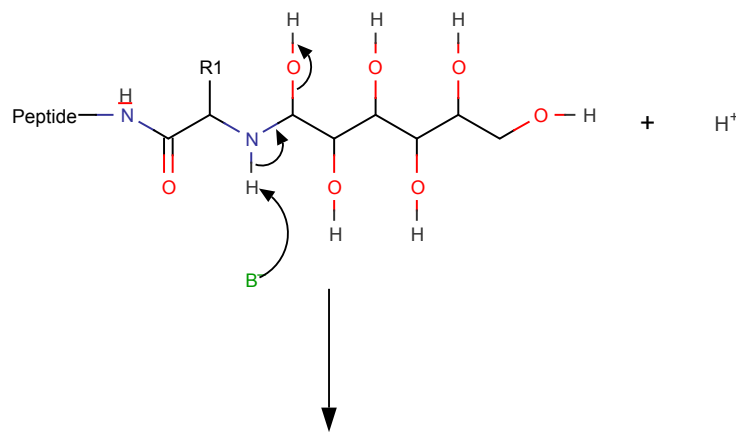

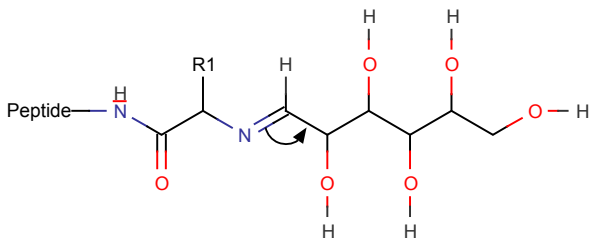

Schiff Base

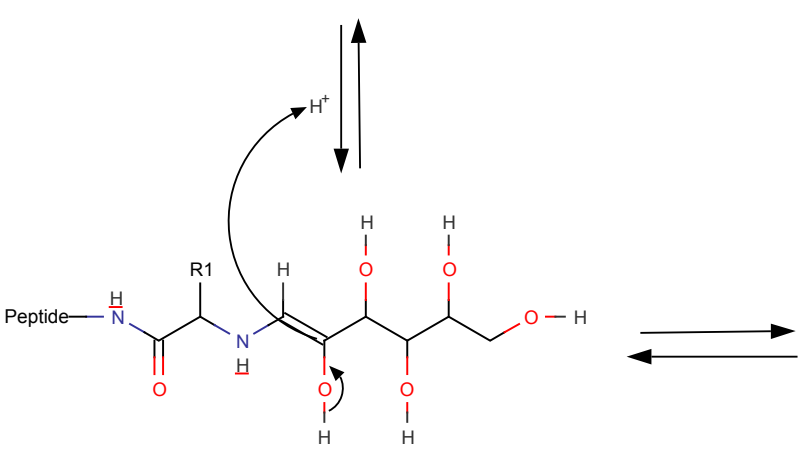

Enol<smiles>CC(C)CNC(=O)C(Br)NC(=O)C(O)C(O)CO</smiles>

Keto

Figure 13: Maillard reaction mechanism.

\section{APIs (drug substances)}

Deamidation is the main degradation pathway for Asn and Glu to produce Asp and Gln, respectively [91]. The reaction is acid-catalyzed and thus pH-dependent [89]. First, the side chain of the amide bond is hydrolysed, followed by nucleophilic attack of water on the amide carbon [89]. In neutral to basic conditions, deamidation proceeds by intramolecular nucleophilic attack of the backbone amide nitrogen on the side-chain amide carbon, producing a tetrahedral intermediate, which degrades to a cyclic imide [89,97]. Figure 12 presents the reaction mechanism for the deamidation reaction of Asn to Asp.

Peptide bond cleavage constitutes a second common degradation mechanism. This is often observed after Asp residues, where an intramolecular attack of the Asp side chain on the C-terminal amide nitrogen to form a cyclic anhydride. Although delocalization of the electrons is possible, this cyclic anhydride is unstable and is hydrolysed to a C-terminal aspartic acid residue $[89,98]$. 


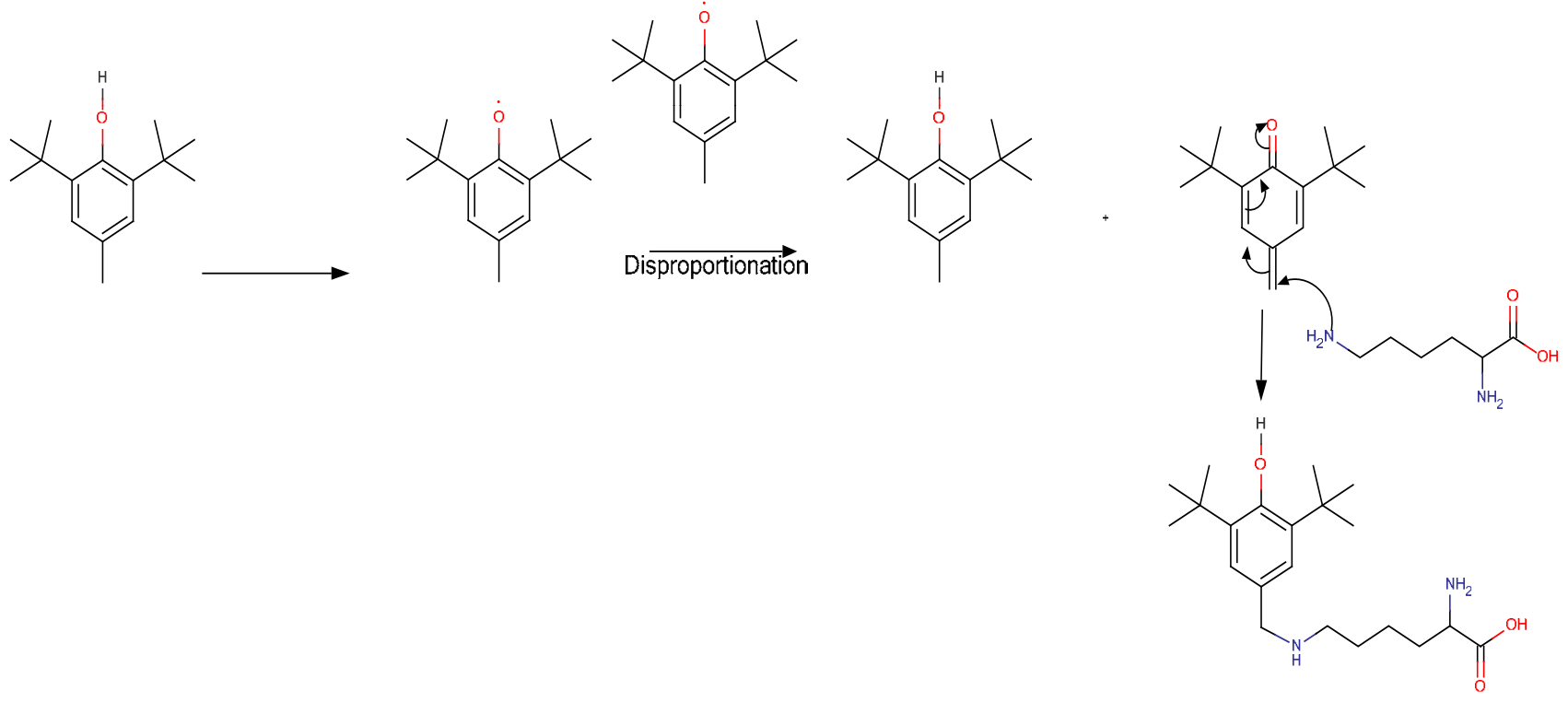

Figure 14: Side-reaction with anti-oxidant BHT.

The side chains of Met, Cys, Trp and Tyr residues are susceptible to oxidation. The mechanism consists of the formation of a Schiff base, followed by tautomerization and hydrolytic cleavage to generate the aldehyde derivative [91].

When reducing sugars (e.g. lactose) are present, these molecules may react with amino or free amine groups via the Maillard reaction. Again, this converts to a Schiff base, which cyclizes and isomerizes as illustrated in Figure 13. This reaction is usually observed for Lys that reacts the most rapidly with reducing sugars, but also for Arg, Asn and Gln $[67,91,99]$.

$\boldsymbol{\beta}$-elimination of disulfide bonds leads to free thiol groups. Basecatalyzed cleavage of the C-S bond in Cys results in dehydroalanine and thiocysteine, which both degrade further. Dehydroalanine is unstable and susceptible to electrophilic addition reactions, whereas thiocysteine degrades to free thiol groups $[91,100,101]$.

\section{FDPs (drug products)}

Although excipients may be added to protect the peptide, they have other properties as well and may cause unwanted degradation by forming interaction products. Of course, the same reactions as explained for the peptide drug substance may be observed in the formulation depending on the storage conditions (moisture, temperature, $\mathrm{pH}$ ), but may be influenced by the micro-environment of the excipients.

Oxidation is known to be metal-catalyzed. In order to reduce this side-reaction, anti-oxidants are added to the formulation, but in turn these may induce reactions as well. For example, the antioxidant 2,6-di-t-butyl-4-methylphenol (BHT) (1) can be oxidized to the corresponding phenoxyl free radical (2), which is able to rapidly disproportionate to produce the corresponding quinine methide (3), which is strongly electrophilic and expected to be reactive with nucleophilic groups like the free amine groups at the $\mathrm{N}$-terminus of the peptide (Figure 14) [102,103].
Formation of hydroperoxides from polyethylene glycol (PEG) in the coating causes electrophilic oxidation. In polymerization processes for polymeric ethers, such as PEG, peroxides are present as initiators, which may induce oxidation of the drug substance during formulation development. This way, oxidation of Met resulted in the formation of Met-sulfoxide [67]. Moreover, impurities present in the excipients are found to be responsible for the enhanced oxidative degradation [66]. In lyophilized peptide formulations, sugars are the most commonly used excipients, but they are known to react with Gly and Arg via the Maillard reaction [91]. In addition, polymeric excipients are currently investigated as release-modifying excipients, but can show chemical interactions with the incorporated peptides. Poly(D,Llactide-co-glycolide) (PLG), poly(lactic acid) (PLA) or poly(D,L-lactic acid-co-glycolic acid) (PLGA) microspheres are used to encapsulate peptides [91]. However, the polyester produces lactic and glycolic acid monomers due to hydrolysis. This way, carboxylic acid is accumulated and creates an acidic microclimate, initiating acid-catalysed peptide degradation [89]. Acylation is a typical example of peptide degradation in polymers. Here, peptides react as a nucleophile with the ester bond of the polymer forming a tetrahedral intermediate, which is further rearranged to produce the acylated peptide [89,104-108]. Previously, we optimized the sample preparation steps for salmon calcitonin, including the development of a complete quality control and stabilityindicating HPLC method for a polymeric calcitonin formulation. Although a temperature-decrease was observed for salmon calcitonin, no degradation products were found, suggesting possible chemical interaction with the polymer [93].

In addition, racemisation may occur when exposed to heat and alkali, involving the removal of the $\alpha$-proton of an amino acid, forming a planar carbanion. The presence of racemised amino acids cannot be revealed with ESI-MS seen there is no mass difference, but enzymatic digestion in combination with MALDI may resolve the identity [109]. 


\section{Conclusion}

Depending on the peptide sequence, different synthesis methodologies can be applied. Because solid-phase is the standard method to synthesize peptides, the different synthesis steps consists of subsequent protecting, coupling and cleavage from the resin. During these reactions steps, additional reagents are added, which may remain present in the final product. Most chemical reactions are not completely finished, thus it is clear that protecting or coupling additives remain attached to the peptide. Moreover, the impurities may be biomedically active or even alter the peptide's activity. So, it is important to analyze the peptide purity by sensitive analytical techniques to make sure the conclusions are correctly interpreted and not due to impurities. Therefore, the different regulatory associations defined acceptance criteria to assure the quality and safety of a peptide drug. In order to assure the quality of peptide drugs, the specifications should meet the requirements as described in the pharmacopoeias. Although, purification steps are generally included after synthesis, we showed that still impure peptides are delivered. In addition, chemical degradation of peptides substances as well as finalized drug products is observed. Although, excipients are added to avoid degradation, this process may still occur or interaction products are produced. So, it is crucial to investigate and report the total impurity and degradant profile of a peptide drug before starting and interpreting biomedical analyses.

\section{Acknowledgments}

This research was funded by a PhD grant of "Institute for the Promotion of Innovation through Science and Technology in Flanders (IWT-Vlaanderen)" 73402 (SVD) as well as by the BOF grant 01J22510 (EW and BDS)

\section{References}

1. Mason JM (2010) Design and development of peptides and peptide mimetics as antagonists for therapeutic intervention. Future Med Chem 2: 1813-1822.

2. Sato AK, Viswanathan M, Kent RB, Wood CR (2006) Therapeutic peptides: Technological advances driving peptides into development. Curr Opin Biotechnol 17: 638-642.

3. Pernot M, Vanderesse R, Frochot C, Guillemin F, Barberi-Heyob M (2011) Stability of peptides and therapeutic success in cancer. Expert Opin Drug Met 7: 793-802.

4. Pollaro L, Heinis C (2010) Strategies to prolong the plasma residence time of peptide drugs. Med Chem Comm 1: 319-324.

5. De Spiegeleer B, Vergote V, Pezeshki A, Peremans K, Burvenich C (2008) Impurity profiling quality control testing of synthetic peptides using liquid chromatography-photodiode array-fluorescence and liquid chromatographyelectrospray ionization-mass spectrometry: The obestatin case. Anal Biochem 376: 229-234.

6. Vergote V, Burvenich C, Van de Wiele C, De Spiegeleer B (2009) Quality specifications for peptide drugs: A regulatory-pharmaceutical approach. J Pept Sci 15: 697-710.

7. Antonioli P, Fortis F, Guerrier L, Rinalducci S, Zolla L, et al. (2007) Capturing and amplifying impurities from purified recombinant monoclonal antibodies via peptide library beads: A proteomic study. Proteomics 7: 1624-1633.

8. de Beukelaar JW, Gratama JW, Smitt PAS, Verjans GM, Kraan J, et al.(2007) The impact of impurities in synthetic peptides on the outcome of t-cell stimulation assays. Rapid Commun Mass Spectrom 21: 1282-1288.

9. Currier JR, Galley LM, Wenschuh H, Morafo V, Ratto-Kim S, et al. (2008) Peptide impurities in commercial synthetic peptides and their implications for vaccine trial assessment. Clin Vaccine Immunol 15: 267-276.

10. Verbeken M, Wynendaele E, Lefebvre R, Goossens E, De Spiegeleer B (2011) The influence of peptide impurity profiles on functional tissue-organ bath response: The 11-mer peptide INSL6 [151-161] case. Anal Biochem do 10.1016/j.ob.2011.09.031.
11. Bari SB,Kadam BR, Jaiswa YS, Shirkhedkar AA (2007) Impurity profile: Significance in active pharmaceuticalingredient. Eurasian J Anal Chem 2: 32 53.

12. Nicolas EC, Scholz TH (1998) Active drug substance impurity profiling - part I. LC/UV diode array spectral matching. J Pharm Biomed Anal 16: 813-824.

13. Roy J (2002) Pharmaceutical impurities- a mini-review. AAPS Pharm Sci Tech 3: $1-8$

14. Baert B, Annavarapu S, Burvenich C, De Spiegeleer B (2009) Analytical biopharmaceutical and regulatory evaluation of topical testosterone preparations. Eur J Pharm Biopharm 72: 275-281.

15. ICH, Q6A (1999): Specifications: Test procedures and acceptance criteria for new drug products: Chemical substances.

16. ICH, Q3A (r2) (2006): Impurities in new drug substances.

17. ICH, Q3B (r2) (2006): Impurities in new drug products.

18. European Pharmacopoeia, Monograph 01/2008:2034 Substances for pharmaceutical use. (2007) (6thedn).

19. European Pharmacopoeia, Monograph 01/2008:51000. (2007). (2001) Contro of impurities in substances for pharmaceutical use. (6thedn).

20. Directive 2001/83/EC of the European parliament and of the Council of 6 November 2001 on the community code relating to medicinal products for human use.

21. European Pharmacopoeia, Monograph 07/2011:1077 (2011) Buserelin (7thedn).

22. European Pharmacopoeia, Monograph 01/2008:2034 (2007) Substances for pharmaceutical use. (6thedn).

23. ICH, Q9 (2006) : Quality risk management.

24. ICH, Q8 (2009): Pharmaceutical development.

25. ICH, Q10 (2009): Pharmaceutical quality system.

26. ICH, Q11 (2011): Development and manufacture of drug substances.

27. FDA, 21CRF58 (1987): Good laboratory practice regulations.

28. OECD principles on good laboratory practice (1998) ENV/MC/Chem (98) 17

29. Guzman F, Barberis S, Illanes A (2007) Peptide synthesis: Chemical or enzymatic. Electron J Biotechnol 10: 279-314.

30. Merrifield RB (1963) Solid phase peptide synthesis - Synthesis of a tetrapeptide J Am Chem Soc 85: 2149-2154.

31. Head DB, Dong JZ, Burton JA (2005) Use of the excluded protecting group (EPG) method for peptide synthesis. J Pept Res 65: 384-394.

32. Hojo K, Maeda M, Kawasaki K (2001) A new water-soluble n-protecting group, 2-(phenyl(methyl)sulfonio)ethyloxycarbonyltetrafluoroborate, and its application to solid phase peptide synthesis in water.J Pept Sci 7: 615-618.

33. Eifler-Lima VL, Graebin CS, Uchoa FD, Duarte PD, Correa AG (2010) Highlights in the solid-phase organic synthesis of natural products and analogues. J Braz Chem Soc 21: 1401-1423.

34. Felgenhauer T, Schirwitz C, Breitling F (2011) Peptide synthesis. In: Ullmann's Encyclopedia of Industrial Chemistry (7thedn), Wiley VCH, Weinheim.

35. Sharma S, Pasha S (1997) Rapid anchoring of cesium salts of Boc-protected amino acids on chloromethyl polystyrene resin using dibenzo-18-crown-6 as a catalyst. Bioorg Med Chem Lett 7: 2077-2080.

36. Lee HJ, Ramaraj B, Yoon KR (2009) Esterification on solid support by surfaceinitiated ring-opening polymerization of epsilon-caprolactone from benzylic hydroxyl-functionalized Wang resin bead. J Appl Polym Sci 111: 839-844.

37. Alewood D, Hopping G, Brust A, Reid RC, Alewood PF (2010) Benzhydrylamine linker grafting: $A$ strategy for the improved synthesis of c-terminal peptide amides. J Pept Sci 16: 551-557.

38. Mezo G, Majer Z, Valero ML, Andreu D, Hudecz F (1999) Synthesis of cyclic herpes simplex virus peptides containing 281-284 epitope of glycoprotein d-1 in endo- or exo-position. J Pept Sci 5: 272-282

39. Mourtas S, Katakalou C, Nicolettou A, Tzavara C, Gatos D, et al. (2003) Resinbound aminothiols: Synthesis and application.Tetrahedron Lett 44: 179-182. 
40. Stathopoulos P, Papas S, Tsikaris V (2006) C-terminal n-alkylated peptide amides resulting from the linker decomposition of the rink amide resin. A new cleavage mixture prevents their formation. J Pept Sci 12: 227-232.

41. Katayama H, Utsumi T, Ozawa C, Nakahara Y, Hojo H, et al. (2009) Pyruvoyl, a novel amino protecting group on the solid phase peptide synthesis and the peptide condensation reaction. Tetrahedron Lett 50: 818-821.

42. Nishio H, Nishiuchi Y, Inui T, Yoshizawa-Kumagaye K, Kimura T (2000) Problems associated with use of the $\mathrm{N}$-in-cyclohexyloxycarbonyl $(\mathrm{Hoc})$ group for tryptophans. Tetrahedron Lett 41: 6839-6842.

43. Wahlstrom K, Unden A (2009) A new protecting group for tryptophan in solidphase peptide synthesis which protects against acid-catalyzed side reactions and facilitates purification by HPLC.Tetrahedron Lett 50: 2976-2978

44. Flemer S (2011) Selenol protecting groups in organic chemistry: Special emphasis on selenocysteineSe-protection in solid phase peptide synthesis. Molecules 16: 3232-3251.

45. Isidro-Llobet A, Alvarez M, Albericio F (2009) Amino acid-protecting groups. Chem Rev 109: 2455-2504.

46. Carpino LA (1993) 1-hydroxy-7-azabenzotriazole - an efficient peptide coupling additive. J Am Chem Soc 115: 4397-4398.

47. Carpino LA, Elfaham A, Minor CA, Albericio F (1994) Advantageous applications of azabenzotriazole (triazolopyridine)-based coupling reagents to solid-phase peptide-synthesis. J Chem Soc, Chem Commun 201-203.

48. Coste J, Lenguyen D, Castro B (1990) PyBOP - a new peptide coupling reagen devoid of toxic by-product.Tetrahedron Lett 31: 205-208.

49. Brown E, Sheppard RC, Williams BJ (1983) Peptide-synthesis. Part4: Solidphase syntheses of peptides related to gastrin. J Chem Soc, Perkin Trans 1 : 75-82.

50. KatritzkyAR, Suzuki K, Singh SK (2004) N-acylation in combinatorial chemistry. ARKIVOC 1: 12-35

51. Valeur E, Bradley M (2009) Amide bond formation: Beyond the myth of coupling reagents. Chem Soc Rev 38: 606-631.

52. Coin I (2010) The depsipeptide method for solid-phase synthesis of difficult peptides. J Pept Sci 16: 223-230.

53. Kroten MA, Bartoszewicz M, Swiecicka I (2010) Cereulide and valinomycin, two important natural dodecadepsipeptides with ionophoretic activities. Pol J Microbiol 59: 3-10.

54. Clark RJ, Craik DJ (2010) Native chemical ligation applied to the synthesis and bioengineering of circular peptides and proteins. Biopolymers $94: 414-422$

55. Tsuji K, Shigenaga A, Sumikawa Y, Tanegashima K, Sato K, et al. (2011) Application of $\mathrm{N}-\mathrm{C}$ - or $\mathrm{C}-\mathrm{N}$ - directed sequential native chemical ligation to the preparation of CXCL14 analogs and their biological evaluation. Bioorg Med Chem 19: 4014-4020.

56. Matsushita T, Hinou H, Kurogochi M, Shimizu H, Nishimura SI (2005) Rapid microwave-assisted solid-phase glycopeptide synthesis. Org Lett 7: 877-880.

57. Santagada V, Frecentese F, Perissutti E, Fiorino F, Severino B, et al. (2009) Microwave assisted synthesis: A new technology in drug discovery. Mini-Rev Med Chem 9: 340-358

58. Bacsa B, Desai B, Dibo G, Kappe CO (2006) Rapid solid-phase peptide synthesis using thermal and controlled microwave irradiation. J Pept Sci 12 : $633-638$

59. Doukyu N, Ogino H (2010) Organic solvent-tolerant enzymes. Biochem Eng J 48: $270-282$

60. Nuijens T, Cusan C, van Dooren T, Moody HM, Merkx R, et al.(2010) Fully enzymatic peptide synthesis using C-terminal tert-Butyl ester interconversion. Adv Synth Catal 352: 2399-2404.

61. Smits R, Koksch B (2006) How C-alpha-fluoroalkyl amino acids and peptides interact with enzymes: Studies concerning the influence on proteolytic stability, enzymatic resolution and peptide coupling. Curr Top Med Chem 6: 1483-1498.

62. Devaskar SU, Giddings SJ, Rajakumar PA, Carnaghi LR, Menon RK, et al (1994) Insulin gene-expression and insulin synthesis in mammalian neuronal cells. J Biol Chem 269: 8445-8454.
63. Johnson IS (1983) Human insulin from recombinant DNA technology. Science 219: 632-637.

64. Bichsel VE, Curcio V, Gassmann R, Otto H (1996) Requirements for the quality control of chemically synthesized peptides and biotechnologically produced proteins. Pharm Acta Helv 71: 439-446.

65. Ermer J (1998) The use of hyphenated LC-MS technique for characterisation of impurity profiles during drug development. J Pharm Biomed Anal 18: 707-714.

66. DiFeo TJ (2004) Safety and efficacy: The role of chemistry, manufacturing, and controls in pharmaceutical drug development. Drug Devind Pharm 30: 247257.

67. Pan C, Liu F, Motto M (2011) Identification of pharmaceutical impurities in formulated dosage forms. J Pharm Sci 100: 1228-1259.

68. Fields GB, Angeletti RH, Carr SA, Smith AJ, Stults JT, et al. (1994) Variable success of peptide-resin cleavage and deprotection following solid-phase synthesis. Tech Protein Chem V, [Pap Symp Protein Soc]: 501-507.

69. Ridge S, Hettiarachchi K (1998) Peptide purity and counter ion determination of bradykinin by highperformance liquid chromatography and capillary electrophoresis. J Chromatogr A 817: 215-222.

70. Gairi M, Lloydwilliams P, Albericio F, Giralt E (1990) Use of BOP reagent for the suppression of diketopiperazine formation in Boc/Bzl solid-phase peptidesynthesis. Tetrahedron Lett 31: 7363-7366.

71. Dolling R, Beyermann M, Haenel J, Kernchen F, Krause E, et al. (1994 Piperidine-mediated side product formation for Asp(Obu(t))-containing peptides. J Chem Soc Chem Commun 853-854

72. Quibell M, Owen D, Packman LC, Johnson T (1994) Suppression of piperidinemediated side product formation for $\mathrm{Asp}(\mathrm{Obu}(\mathrm{t}))$-containing peptides by the use of $\mathrm{N}$-(2-hydroxy-4-methoxybenzyl) (Hmb) backbone amide protection. J Chem Soc Chem Commun 2343-2344.

73. Yang Y, Sweeney WV, Schneider K, Thornqvist S, Chait BT, et al. (1994) Aspartimide formation in base-driven 9-fluorenylmethoxycarbonyl chemistry. Tetrahedron Lett 35: 9689-9692.

74. Gairi M, Lloydwilliams P, Albericio F, Giralt E (1994) Severe side-reaction in the acidolytic cleavage of a C-terminal Met-containing peptide from the solid support - formation of the homoserine lactone peptide. Tetrahedron Lett 35 175-178.

75. Sakakibara S, Hess GP, Shin KH (1962) An approach to specific cleavage of peptide bonds - Acyl migration in dipeptides containing hydroxyamino acids in anhydrous hydrogen fluoride. J Am Chem Soc 84: 4921-4928

76. Mouls L, Subra G, Enjalbal C, Martinez J, Aubagnac JL (2004) O-N-acy migration in $\mathrm{N}$-terminal serine-containing peptides: Mass spectrometric elucidation and subsequent development of site-directed acylation protocols Tetrahedron Lett 45: 1173-1178.

77. Sohma Y, Hayashi Y, Skwarczynski M, Hamada Y, Sasaki M, et al. (2004) O-Nintramolecular acyl migration reaction in the development of prodrugs and the synthesis of difficult sequence-containing bioactive peptides. Biopolymers 76: 344-356.

78. Feinberg RS, Merrifield RB (1975) Modification of peptides containing glutamicacid by hydrogen fluoride-anisole mixtures - gamma-acylation of anisole or glutamyl nitrogen. J Am Chem Soc 97: 3485-3496.

79. Wu CR, Wade JD, Tregear GW (1988) Beta-subunit of baboon chorionic gonadotropin - continuous-flow Fmoc-polyamide synthesis of the C-terminal 37-peptide. Int J Pept Protein Res 31: 47-57.

80. Li N, Fort F, Kessler K, Wang W (2009) Factors affecting cleavage at aspartic residues in model decapeptides. J Pharm Biomed Anal 50: 73-78.

81. Karlstrom A, Unden A (1996) A new protecting group for aspartic acid that minimizes piperidine-catalyzedaspartimide formation in Fmoc solid phase peptide synthesis. Tetrahedron Lett 37: 4243-4246.

82. Obkircher M, Stahelin C, Dick F (2008) Formation of Fmoc-beta-alanine during fmoc-protections with Fmoc-Osu. J Pept Sci 14: 763-766.

83. Papayannopoulos IA (1996) Use of low- and high-energy collision-induced dissociation tandem mass spectrometry in the identification of an unusual amino acid in a semisynthetic polypeptide. J Am Soc Mass Spectrom 7: 1034 1039. 
Citation: Van Dorpe S, Verbeken M, Wynendaele E, De Spiegeleer B (2011) Purity Profiling of Peptide Drugs. J Bioanal Biomed S6: 003. doi:10.4172/1948-593X.S6-003

84. Pennington MW (1994) Hf cleavage and deprotection procedures for peptides synthesized using a Boc/Bzl strategy. In: Peptide Synthesis Protocols, Humana Press, Totowa.

85. Gomes I, Grushko JS, Golebiewska U, Hoogendoorn S, Gupta A, et al. (2009) Novel endogenous peptide agonists of cannabinoid receptors. FASEB J 23: 3020-3029.

86. Kopchick JJ, Parkinson C, Stevens EC, Trainer PJ (2002) Growth hormone receptor antagonists: Discovery, development, and use in patients with acromegaly. Endocr Rev 23: 623-646.

87. de Beukelaar JW, Gratama JW, Smitt PAS, Verjans GM, Kraan J, et al.(2007) The impact of impurities in synthetic peptides on the outcome of T-cel stimulation assays. Rapid Commun Mass Spectrom 21: 1282-1288.

88. Metzger JW, Kempter C, Wiesmuller KH, Jung G (1994) Electrospray massspectrometry and tandem mass-spectrometry of synthetic multicomponent peptide mixtures - determination of composition and purity. Anal Biochem 219: 261-277.

89. Houchin ML, Topp EM (2008) Chemical degradation of peptides and proteins in PLGA: A review of reactions and mechanisms. J Pharm Sci 97: 2395-2404.

90. De Boni S, Oberthur C, Hamburger M, Scriba GKE (2004) Analysis of aspartyl peptide degradation products by high-performance liquid chromatography and high-performance liquid chromatography-mass spectrometry. J Chromatogr A 1022: 95-102.

91. Lai MC, Topp EM (1999) Solid-state chemical stability of proteins and peptides.J Pharm Sci 88: 489-500

92. D'Hondt M, Demare W, Van Dorpe S, Wynendaele E, Burvenich C, et al.(2011) Dry heat stress stability evaluation of casein peptide mixture. Food Chem 128 : 114-122.

93. D'Hondt M, Van Dorpe S, Mehuys E, Deforce D, De Spiegeleer B (2010) Quality analysis of salmon calcitonin in a polymeric bioadhesive pharmaceutical formulation: Sample preparation optimization by doe. J Pharm Biomed Anal 53 : 939-945.

94. Rothen-Weinhold A, Oudry N, Schwach-Abdellaoui K, Frutiger-Hughes S, Hughes GJ, et al. (2000) Formation of peptide impurities in polyester matrices during implant manufacturing. Eur J Pharm Biopharm 49: 253-257.

95. Jorgensen L, Hostrup S, Moeller EH, Grohganz H (2009) Recent trends in stabilising peptides and proteins in pharmaceutical formulation - considerations in the choice of excipients. Expert Opin Drug Delivery 6: 1219-1230.

96. DiFeo TJ (2003) Drug product development: A technical review of chemistry, manufacturing, and controls information for the support of pharmaceutical compound licensing activities. Drug Dev Ind Pharm 29: 939-958.

97. Wakankar AA, Borchardt RT (2006) Formulation considerations for proteins susceptible to asparagine deamidation and aspartate isomerization. J Pharm Sci 95: 2321-2336.

98. Wang RH, Feder D, Hsieh F (2003) Characterization of eptifibatide during drug formulation stability assays. J Pharm Biomed Anal 33: 1181-1187.

99. Garbe LA, Wuertz A, Piechotta CT, Tressl R (2008) The peptide-catalyzed Maillard reaction - characterization of $\mathrm{C}-13$ reductones.In: Maillard Reaction Recent Advances in Food and Biomedical Sciences, (1stedn), Wiley-Blackwell, Weinheim

100.Ni S, Zhang H, Huang W, Zhou J, Qian H, et al.The application of an ary hydrazine linker prevents beta-elimination side products in the SPPS of C-terminal cysteine peptides. J Pept Sci 16: 309-313

101. Herbert B, Hopwood F, Oxley D, McCarthy J, Laver M, et al. (2003) Betaelimination: An unexpected artefact in proteome analysis. Proteomics 3: 826831.

102. Feibush B, Snyder BC (2000) Oxidation of the N-terminal Gly-residue of peptides: Stress study of pexigauan acetate in a drug formulation. Pharm Res 17: 197-204.
103. Wu Y, Dali M, Gupta A, Raghavan K (2009) Understanding drug-excipient compatibility: Oxidation of compound a in a solid dosage form. Pharm Dev Technol 14: 556-564.

104. Lucke A, Kiermaier J, Gopferich A (2002) Peptide acylation by poly(alphahydroxy esters). Pharm Res 19: 175-181.

105.Park EJ, Tak TH, Na DH, Lee KC (2010) Effect of PEGylation on stability of peptide in poly(lactide-co-glycolide) microspheres. Arch Pharmacal Res 33 1111-1116.

106. Murty SB, Na DH, Thanoo BC, DeLuca PP (2005) Impurity formation studies with peptide-loaded polymeric microspheres. Part II. In vitro evaluation. Int J Pharm 297: 62-72.

107. Murty SB, Thanoo BC, Wei Q, DeLuca PP (2005) Impurity formation studies with peptide-loaded polymeric microspheres. Part I. In vivo evaluation. Int J Pharm 297: 50-61.

108. Na DH, Lee JE, Jang SW, Lee KC (2007) Formation of acylated growth hormone-releasing peptide- 6 by poly(lactide-co-glycolide) and its biological activity. AAPS Pharm Sci Tech 8.

109. Senderoff RI, Kontor KM, Kreilgaard L, Chang JJ, Patel S, et al. (1998) Consideration of conformational transitions and racemization during process development of recombinant glucagon-like peptide-1. J Pharm Sci 87: 183189

This article was originally published in a special issue, Advances in Drug development handled by Editor(s). Dr. Heng Hong, East Carolina University, USA 